\title{
Sharp Weyl estimates for tensor products of pseudodifferential operators
}

\author{
Ubertino Battisti $^{1}$ - Massimo Borsero ${ }^{1}$. \\ Sandro Coriasco ${ }^{1}$
}

Received: 4 December 2014 / Accepted: 4 March 2015 / Published online: 24 March 2015

(C) Fondazione Annali di Matematica Pura ed Applicata and Springer-Verlag Berlin Heidelberg 2015

\begin{abstract}
We study the asymptotic behavior of the counting function of tensor products of operators, in the cases where the factors are either pseudodifferential operators on closed manifolds or pseudodifferential operators of Shubin type on $\mathbb{R}^{n}$, respectively. We obtain, in particular, the sharpness of the remainder term in the corresponding Weyl formulae, which we prove by means of the analysis of some explicit examples.
\end{abstract}

Keywords Weyl's law · Tensor product of pseudodifferential operators · Bisingular Operators

Mathematics Subject Classification $35 \mathrm{P} 20 \cdot 35 \mathrm{P} 15$

\section{Contents}

1 Introduction . . . . . . . . . . . . . . . . . . . . . . 796

2 Preliminary results . . . . . . . . . . . . . . . . . . . . . . . . . . . 799

3 Spectral asymptotics for the tensor product of two operators . . . . . . . . . . . . . . . . 801

4 Spectral asymptotics for the tensor product of $r$ operators . . . . . . . . . . . . . . 805

5 Sharpness of the result . . . . . . . . . . . . . . . . . . . . . . . . 811

Appendix: The Dirichlet divisors problem . . . . . . . . . . . . . . . . . . . . . . . . 818

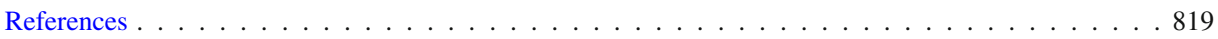

Ubertino Battisti

ubertino.battisti@unito.it

Massimo Borsero

massimo.borsero@unito.it

Sandro Coriasco

sandro.coriasco@unito.it

1 Dipartimento di Matematica 'Giuseppe Peano', Università degli Studi di Torino, Turin, Italy 


\section{Introduction}

Let $P$ be a positive self-adjoint operator of order $m>0$ with domain $H^{m}(M) \hookrightarrow L^{2}(M)$, $M$ a Riemannian, $n$-dimensional smooth closed manifold. Assume that the resolvent of $P$ is compact, so that the spectrum is discrete and given by a sequence of eigenvalues with finite multiplicities. Let $\left\{\lambda_{j}\right\}_{j \in \mathbb{N}}=\sigma(P)$ be the set of the eigenvalues of $P$, repeated according to their multiplicity. The counting function $N_{P}(\tau)$ is defined as

$$
N_{P}(\tau)=\sum_{\lambda_{j} \in \sigma(P) \cap[0, \tau)} 1=\sum_{\lambda_{j}<\tau} 1 .
$$

The Weyl law, see, e.g., $[15,16]$, describes the asymptotic expansion of the counting function $N_{P}(\tau)$, as $\tau$ goes to infinity. It is well known that the leading term of the asymptotic expansion of (1) depends on the dimension of the manifold, on the order of the operator and on its principal symbol, see, e.g., [16]. Similar formulae can be obtained in many other different settings, see [24] and [2] for a detailed analysis and several developments. To mention a few specific situations, see $[18,26]$ for the case of the Shubin calculus on $\mathbb{R}^{n}$, [7] for the anisotropic Shubin calculus, $[5,8,21]$ for the $S G$-operators on $\mathbb{R}^{n}$ and the manifolds with ends, [10] for operators on conic manifolds, [20] for operators on cusp manifolds, [9] for operators on asymptotic hyperbolic manifolds and [4,6] for bisingular operators.

In this paper, we study the counting function of the tensor product of $r$ pseudodifferential operators. We consider the cases of Hörmander operators on closed manifolds and of the Shubin calculus on $\mathbb{R}^{n}$. In the case $r=2$, for classical Hörmander operators on closed manifolds, the operators we consider are a subclass of the so-called bisingular operators, studied by Rodino in [23] (see also [22]) in connection with the multiplicative property of the AtiyahSinger index [3]. An asymptotic expansion of the counting function of bisingular operators was obtained by the first author in [4]. The basic tool was the spectral $\zeta$-function, in the spirit of Guillemin's so-called soft proof of the Weyl law [13]. This method allows determining the leading term of the asymptotic expansion in the non-symmetric case (corresponding to a simple first pole of the spectral $\zeta$-function). In the symmetric case, the spectral $\zeta$-function has a first pole of order 2. Using a theorem due to Aramaki [1], it has been possible to determine the leading term, which has a behavior of type $\tau^{p} \log \tau$, as well as the second term, which has a behavior of type $\tau^{p}, p$ being the first pole of the spectral $\zeta$-function. However, it was not possible, through the aforementioned method, to give a good estimate of the remainder term. We notice that the asymptotic behavior of the counting function in the bisingular case has some similarities with the Weyl law in the setting of $S G$-classical operators on manifolds with ends $[5,8]$.

A version of bisingular operators, based on Shubin pseudodifferential calculus on $\mathbb{R}^{n}$, was introduced in [6]. The counting function was studied also in this setting, obtaining results analogous to those which hold for the "standard" bisingular calculus.

In this paper, we consider the same class of operators studied in [11], namely, tensor products of $r$ pseudodifferential operators, that is,

$$
A=A_{1} \otimes \cdots \otimes A_{r} .
$$

In the sequel, we will assume either that each $A_{j}$ is a classical Hörmander pseudodifferential operator on a $n_{j}$-dimensional closed manifolds $M_{j}$, that is, $A_{j} \in L_{\mathrm{cl}}^{m_{j}}\left(M_{j}\right), j=1, \ldots, r$, or that each $A_{j}$ belongs to a classical global Shubin class on $\mathbb{R}^{n_{j}}$, that is, $A_{j} \in G_{\mathrm{cl}}^{m_{j}}\left(\mathbb{R}^{n_{j}}\right)$, $j=1, \ldots, r$. We also assume that $A$ is positive, self-adjoint and Fredholm. It is straightforward to check that the Fredholm property of $A$ implies that $A_{j}$ is invertible for any 
$j=1, \ldots, r$. We illustrate here our results in the case $r=2$, see Sect. 4 below for the statements which hold for an arbitrary number of factors.

Denoting by $\sigma\left(A_{1}\right)=\left\{\lambda_{j}\right\}_{j \in \mathbb{N}}$ and $\sigma\left(A_{2}\right)=\left\{\mu_{k}\right\}_{k \in \mathbb{N}}$ the spectra of $A_{1}$ and $A_{2}$, with eigenvalues repeated according with their multiplicities, we easily obtain that the spectrum of $A$ is given by

$$
\sigma(A)=\left\{\lambda_{j} \cdot \mu_{k}\right\}_{(j, k) \in \mathbb{N}^{2}}
$$

Therefore,

$$
N_{A}(\tau)=\sum_{\rho \in \sigma(A) \cap[0, \tau)} 1=\sum_{\lambda_{j} \cdot \mu_{k}<\tau} 1 .
$$

Assume that $A=A_{1} \otimes A_{2}$ is positive, self-adjoint and Fredholm, with $A_{1} \in L_{\mathrm{cl}}^{m_{1}}\left(M_{1}\right)$, $A_{2} \in L_{\mathrm{cl}}^{m_{2}}\left(M_{2}\right), m_{1}, m_{2}>0, \operatorname{dim} M_{1}=n_{1}, \operatorname{dim} M_{2}=n_{2}$ and $\frac{n_{1}}{m_{1}}>\frac{n_{2}}{m_{2}}$. Our first main result, proved in Theorem 2, states that, under such assumptions,

$$
N_{A}(\tau)= \begin{cases}\frac{C_{1}}{n_{1}} \zeta\left(A_{2}, \frac{n_{1}}{m_{1}}\right) \tau^{\frac{n_{1}}{m_{1}}}+\mathcal{O}\left(\tau^{\frac{n_{1}-1}{m_{1}}}\right) & \text { if } \frac{n_{2}}{m_{2}}<\frac{n_{1}-1}{m_{1}} \\ \frac{C_{1}}{n_{1}} \zeta\left(A_{2}, \frac{n_{1}}{m_{1}}\right) \tau^{\frac{n_{1}}{m_{1}}}+\mathcal{O}\left(\tau^{\frac{n_{1}-1}{m_{1}}} \log \tau\right) & \text { if } \frac{n_{2}}{m_{2}}=\frac{n_{1}-1}{m_{1}} \\ \frac{C_{1}}{n_{1}} \zeta\left(A_{2}, \frac{n_{1}}{m_{1}}\right) \tau^{\frac{n_{1}}{m_{1}}}+\mathcal{O}\left(\tau^{\frac{n_{2}}{m_{2}}}\right) & \text { if } \frac{n_{2}}{m_{2}}>\frac{n_{1}-1}{m_{1}}\end{cases}
$$

for $\tau \rightarrow+\infty$. In (3), $\zeta$ denotes the spectral $\zeta$-function and

$$
C_{1}=\frac{1}{(2 \pi)^{n_{1}}} \int_{M_{1}} \int_{\mathbb{S}^{n_{1}-1}} \frac{\mathrm{d} \theta_{1} \mathrm{~d} x_{1}}{\left[a_{m_{1}}\left(x_{1}, \theta_{1}\right)\right]^{\frac{n_{1}}{m_{1}}}} .
$$

A similar statement holds for the tensor product of two Shubin operators with positive order. Moreover, using spherical harmonics, we show that the estimate (3) is sharp.

In [11], Gramchev, Pilipović, Rodino and Vindas considered the same class of operators, finding a slightly weaker estimate for the remainder term of the Weyl formula. Explicitly, they prove that, under the assumptions stated above,

$$
N_{A}(\tau)=\frac{C_{1}}{n_{1}} \zeta\left(A_{2}, \frac{n_{1}}{m_{1}}\right) \tau^{\frac{n_{1}}{m_{1}}}+\mathcal{O}\left(\tau^{\delta}\right)
$$

where $\max \left\{\frac{n_{1}-1}{m_{1}}, \frac{n_{2}}{m_{2}}\right\}<\delta<\frac{n_{1}}{m_{1}}$.

The asymptotic expansion in (3) is related with the position of the first poles of the spectral $\zeta$-function associated with $A_{1}$ and $A_{2}$, as sketched in the following pictures. 

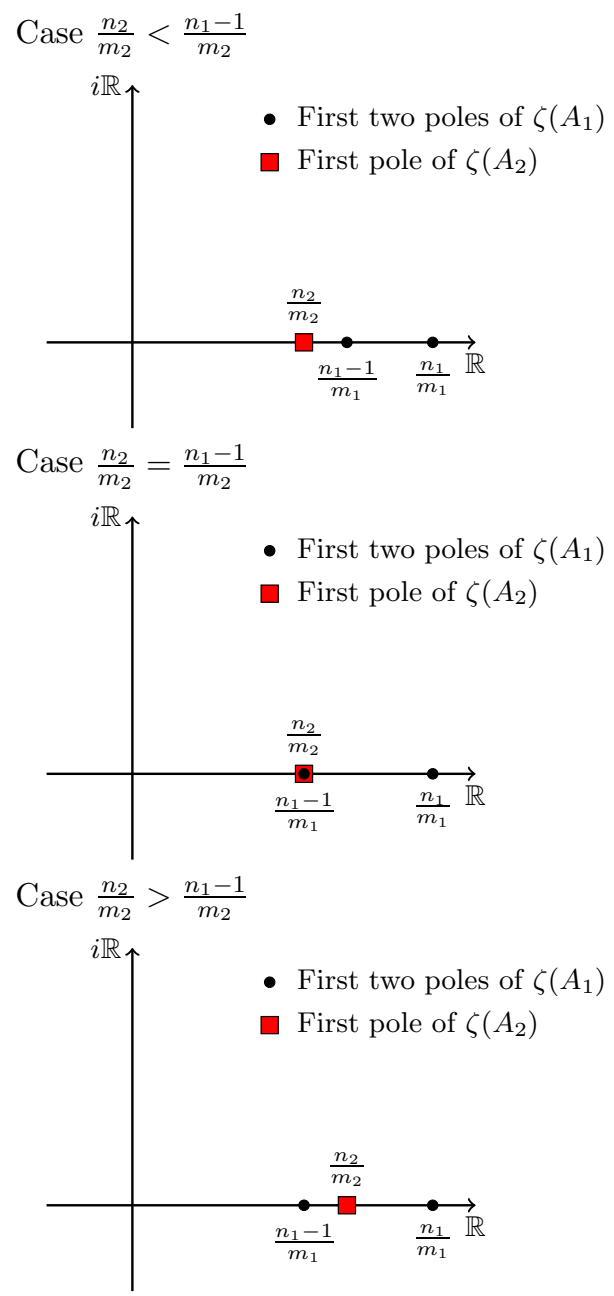

The key point in the proof of our results is the following equivalence, explained in (13):

$$
N_{A}(\tau)=\sum_{\lambda_{j} \cdot \mu_{k}<\tau} 1=\sum_{\mu_{k}<\tau} N_{A_{2}}\left(\frac{\tau}{\mu_{k}}\right) .
$$

The argument is then a careful application of the well-known sharp Weyl law. A main aspect is the possibility to estimate the reminder term, in the Weyl law of $A_{2}$ evaluated in $\frac{\tau}{\mu_{k}}$, uniformly with respect to $\mu_{k}$.

The paper is organized as follows. In Sect. 2, we shortly recall the Weyl laws in the case of the Hörmander calculus on closed manifolds and of the Shubin calculus on $\mathbb{R}^{n}$. We also study the asymptotic behavior of the sum

$$
\sum_{\mu_{k}<\tau} \frac{1}{\mu_{k}^{c}}
$$

for different ranges of $c \in \mathbb{R}$, where $\left\{\mu_{k}\right\}_{k \in \mathbb{N}}$ is the spectrum of an operator in the calculus we consider. In Sect. 3, we prove our main results in the case of tensor products of two 
factors. In Sect. 4, we extend the results to the case of tensor products of $r>2$ factors. In Sect. 5, we show that our estimates of the remainder term of the Weyl law are sharp, focusing again on the case of tensor products of two factors. Finally, we collect in the Appendix some remarks concerning the connection of this analysis with lattice problems, in particular with the Dirichlet divisor problem in the classic setting and in the anisotropic case.

\section{Preliminary results}

We recall well-known results on the sharp Weyl law in the case of operators on closed manifolds and of operators of Shubin type on $\mathbb{R}^{n}$, see, e.g., Hörmander [15], Hellfer and Robert [18], see also [17].

Theorem 1 (Sharp Weyl law) Let A be a positive self-adjoint elliptic classical pseudodifferential operator in $L_{\mathrm{cl}}^{m}(M)$, with $M$ a closed manifold of dimension n, and let $\sigma(A)=\left\{\lambda_{j}\right\}_{j \in \mathbb{N}}$ be its spectrum. Then,

$$
N_{A}(\lambda)=\sum_{\lambda_{j}<\lambda} 1=\frac{C_{A}}{n} \lambda^{\frac{n}{m}}+R_{A}(\lambda)
$$

where

$$
C_{A}=\frac{1}{(2 \pi)^{n}} \int_{M} \int_{\mathbb{S}^{n-1}} \frac{d \theta d x}{\left[a_{m}(x, \theta)\right]^{\frac{n}{m}}},
$$

with $a_{m}$ the principal homogeneous symbol of $A$, and

$$
\limsup _{\lambda \rightarrow+\infty} \frac{\left|N_{A}(\lambda)-\frac{C_{A}}{n} \lambda^{\frac{n}{m}}\right|}{\lambda^{\frac{n-1}{m}}}=\limsup _{\lambda \rightarrow+\infty} \frac{\left|R_{A}(\lambda)\right|}{\lambda^{\frac{n-1}{m}}}<+\infty .
$$

Analogously, let $P \in G_{\mathrm{cl}}^{m}\left(\mathbb{R}^{n}\right)$ be a positive self-adjoint elliptic classical pseudodifferential operator of Shubin type on $\mathbb{R}^{n}$ with $m>0$, and let $\sigma(P)=\left\{\mu_{k}\right\}_{k \in \mathbb{N}}$ be its spectrum. Then,

$$
N_{P}(\lambda)=\sum_{\mu_{k}<\lambda} 1=\frac{K_{P}}{2 n} \lambda^{\frac{2 n}{m}}+R_{P}(\lambda),
$$

where

$$
K_{P}=\frac{1}{(2 \pi)^{2 n}} \int_{\mathbb{S}^{2 n-1}} \frac{d \theta}{\left[p_{m}(\theta)\right]^{\frac{2 n}{m}}},
$$

with $p_{m}$ the principal homogeneous symbol of $P$, and

$$
\limsup _{\lambda \rightarrow+\infty} \frac{\left|N_{P}(\lambda)-\frac{K_{P}}{2 n} \lambda^{\frac{2 n}{m}}\right|}{\lambda^{\frac{2 n-1}{m}}}=\limsup _{\lambda \rightarrow+\infty} \frac{\left|R_{P}(\lambda)\right|}{\lambda^{\frac{2 n-1}{m}}}<+\infty .
$$

The next Propositions 1 and 2 will be crucial in our proof of the Weyl law with sharp remainder for tensor products. They follow as consequence of well-known properties of the spectra of positive self-adjoint operators. We examine in detail only the case of Hörmander pseudodifferential operators on closed manifold, since the argument for the case of Shubin operators is similar. 
Proposition 1 Let $M$ be a closed manifold of dimension $n$, and $A \in L_{\mathrm{cl}}^{m}(M), m>0$, be elliptic, positive and self-adjoint, with spectrum $\sigma(A)=\left\{\mu_{k}\right\}_{k \in \mathbb{N}}$. Define

$$
F_{A}(\tau, c)=\sum_{\mu_{k}<\tau} \frac{1}{\mu_{k}^{c}}=\left\{\begin{array}{lll}
F_{1}(\tau) & \text { if } & c>\frac{n}{m}, \\
F_{2}(\tau) & \text { if } & c=\frac{n}{m}, \\
F_{3}(\tau) & \text { if } & c<\frac{n}{m} .
\end{array}\right.
$$

Then,

$$
\limsup _{\tau \rightarrow+\infty} \frac{\zeta(A, c)-F_{1}(\tau)}{\tau^{\frac{n}{m}-c}}=\kappa_{1}, \limsup _{\tau \rightarrow+\infty} \frac{F_{2}(\tau)}{\log \tau}=\kappa_{2}, \limsup _{\tau \rightarrow+\infty} \frac{F_{3}(\tau)}{\tau^{\frac{n}{m}-c}}=\kappa_{3},
$$

for suitable positive constants $\kappa_{1}, \kappa_{2}, \kappa_{3}$. That is, for $\tau \rightarrow+\infty$,

$$
\zeta(A, c)-F_{1}(\tau)=\mathcal{O}\left(\tau^{\frac{n}{m}-c}\right), F_{2}(\tau)=\mathcal{O}(\log \tau), F_{3}(\tau)=\mathcal{O}\left(\tau^{\frac{n}{m}-c}\right) .
$$

Proof If $c>\frac{n}{m}$ it is immediate that the series $\sum_{k=0}^{\infty} \frac{1}{\mu_{k}^{c}}$ is convergent, in view of the holomorphic properties of the spectral $\zeta$-function associated with $A$. To prove the asymptotic properties of $\zeta(A, c)-F_{1}(\tau)$, we switch to $B=A^{1 / m}$, so that the order of $B$ is one and $\sigma(B)=\mu_{k}^{1 / m}$. We have

$$
\begin{aligned}
\zeta(A, c)-F_{1}(\tau) & =\sum_{\mu_{k} \geq \tau} \frac{1}{\mu_{k}^{c}}=\sum_{\mu_{k}^{1 / m} \geq \tau^{1 / m}} \frac{1}{\left(\mu_{k}^{1 / m}\right)^{c m}} \\
& =\int_{\tau^{1 / m}}^{+\infty} \frac{1}{\mu^{c m}} \mathrm{~d} N_{B}(\mu) .
\end{aligned}
$$

Since $B$ is of order one, it is well known that

$$
N_{B}(\lambda+1)-N_{B}(\lambda) \leq \sharp\{\sigma(B) \cap[\lambda, \lambda+1]\}=\mathcal{O}\left(\lambda^{n-1}\right), \lambda \rightarrow+\infty
$$

(see, e.g., [12, § 12]). Using (9) and the properties of Stieltjes integral, we obtain, for $\tau \rightarrow$ $+\infty$

$$
\begin{aligned}
\zeta(A, c)-F_{1}(\tau) & =\int_{\tau^{1 / m}}^{+\infty} \frac{1}{\mu^{c m}} \mathrm{~d} N_{B}(\mu) \\
& \leq \sum_{j=\left[\tau^{1 / m}\right]-1}^{\infty} \sup _{\mu \in[j, j+1]}\left(\frac{1}{\mu^{c m}}\right)\left(N_{B}(j+1)-N_{B}(j)\right) \\
& \leq \kappa \sum_{j=\left[\tau^{1 / m}\right]-1}^{\infty} \frac{1}{j^{c m-n+1}} \\
& \leq \kappa \int_{\left[\tau^{1 / m}\right]-1}^{+\infty} \frac{1}{(t-1)^{c m-n+1}} \mathrm{~d} t \\
& =\kappa \frac{1}{c m-n}\left[\tau^{1 / m}-2\right]^{n-m c} \in \mathcal{O}\left(\tau^{\frac{n}{m}-c}\right) .
\end{aligned}
$$

where $[a]$ denotes the minimum integer such that $[a] \geq a$. 
To prove the results for $F_{2}$ and $F_{3}$ we can assume, without loss of generality, that $\mu_{0}=$ $\tilde{\mu}_{0}=1$. Using again the properties of the Stieltjes integral, we write

$$
F_{A}(\tau, c)=\int_{1}^{\tau^{1 / m}} \frac{1}{\mu^{c m}} \mathrm{~d} N_{B}(\mu) \leq \sum_{j=1}^{\left[\tau^{1 / m}\right]} \sup _{\mu \in[j, j+1]}\left(\frac{1}{\mu^{c m}}\right)\left(N_{B}(j+1)-N_{B}(j)\right) .
$$

Let us initially suppose that $c>0$, so that $\frac{1}{x^{c}}$ is a decreasing function on $[1,+\infty)$. In view of (9), we have

$$
\begin{aligned}
& \int_{1}^{\tau^{1 / m}} \frac{1}{\mu^{c m}} \mathrm{~d} N_{B}(\mu) \leq \sum_{j=1}^{\left[\tau^{1 / m}\right]} \frac{1}{j^{c m}} \mathcal{O}\left(j^{n-1}\right) \leq \widetilde{\kappa} \sum_{j=1}^{\left[\tau^{1 / m}\right]} \frac{1}{j^{c m-n+1}} \\
& \leq \widetilde{\kappa}\left(\int_{1}^{\left[\tau^{1 / m}\right]} t^{n-c m-1} \mathrm{~d} t+1\right) .
\end{aligned}
$$

By integration, we find

$$
F_{A}(\tau, c)=\int_{1}^{\tau^{1 / m}} \frac{1}{\mu^{c m}} \mathrm{~d} N_{B}(\mu) \leq\left\{\begin{array}{llc}
\frac{\widetilde{\kappa}_{1}}{n-c m} \tau^{\frac{n}{m}-c} & \text { if } & 0<c<\frac{n}{m}, \\
\frac{\widetilde{\kappa}_{2}}{m} \log \tau & \text { if } & c=\frac{n}{m},
\end{array}\right.
$$

as claimed. Finally, if $c \leq 0$, then $\frac{1}{\mu^{c}}$ is a non-decreasing function and also in this case, similar to (10), we obtain

$$
F_{A}(\tau, c) \leq \kappa \int_{1}^{\left[\tau^{1 / m}\right]}(x+1)^{n-c m-1} \mathrm{~d} x \leq \frac{\widetilde{\kappa}_{3}}{n-c m} \tau^{\frac{n}{m}-c} .
$$

The proof is complete.

Proposition 2 Let $P \in G_{\mathrm{cl}}^{m}\left(\mathbb{R}^{n}\right)$ be an elliptic, positive and self-adjoint Shubin operator of order $m>0$, with spectrum given by $\sigma(P)=\left\{\lambda_{j}\right\}_{j \in \mathbb{N}}$. Define

$$
F_{P}(\tau, c)=\sum_{\lambda_{j}<\tau} \frac{1}{\lambda_{j}^{c}}=\left\{\begin{array}{llc}
F_{1}(\tau) & \text { if } & c>\frac{2 n}{m}, \\
F_{2}(\tau) & \text { if } & c=\frac{2 n}{m} \\
F_{3}(\tau) & \text { if } & c<\frac{2 n}{m}
\end{array}\right.
$$

Then,

$$
\limsup _{\tau \rightarrow+\infty} \frac{\zeta(P, c)-F_{1}(\tau)}{\tau^{\frac{2 n}{m}-c}}=\kappa_{1}, \quad \limsup _{\tau \rightarrow+\infty} \frac{F_{2}(\tau)}{\log \tau}=\kappa_{2}, \quad \limsup _{\tau \rightarrow+\infty} \frac{F_{3}(\tau)}{\tau^{\frac{2 n}{m}-c}}=\kappa_{3},
$$

for suitable positive constants $\kappa_{1}, \kappa_{2}, \kappa_{3}$. That is, for $\tau \rightarrow+\infty$,

$$
\zeta(P, c)-F_{1}(\tau)=\mathcal{O}\left(\tau^{\frac{2 n}{m}-c}\right), F_{2}(\tau)=\mathcal{O}(\log \tau), F_{3}(\tau)=\mathcal{O}\left(\tau^{\frac{2 n}{m}-c}\right) .
$$

\section{Spectral asymptotics for the tensor product of two operators}

We start considering the case of the tensor product of two operators. Let $M_{1}, M_{2}$ be two compact manifolds of dimension $n_{1}, n_{2}$, respectively. Let $A=A_{1} \otimes A_{2}, A_{j} \in L_{\mathrm{cl}}^{m_{j}}\left(M_{j}\right)$, 
$m_{j}>0, j=1,2$. Assume that the spectra of $A_{1}$ and $A_{2}$ are sequences of eigenvalues and set

$$
\sigma\left(A_{1}\right)=\left\{\lambda_{j}\right\}_{j \in \mathbb{N}}, \quad \sigma\left(A_{2}\right)=\left\{\mu_{k}\right\}_{k \in \mathbb{N}}
$$

so that

$$
\sigma(A)=\left\{\lambda_{j} \cdot \mu_{k}: \lambda_{j} \in \sigma\left(A_{1}\right), \mu_{k} \in \sigma\left(A_{2}\right)\right\} .
$$

For simplicity, we start with the case $m_{1}=1$ and $n_{1}>\frac{n_{2}}{m_{2}}$. Let $c$ be an arbitrary positive constant and $B$ an operator with spectrum $\sigma(B)=\left\{\mu_{k}\right\}_{k \in \mathbb{N}}$; then, $\sigma(c B)=\left\{c \cdot \mu_{k}\right\}_{k \in \mathbb{N}}$. There is a simple and useful formula relating the counting functions $N_{c B}$ and $N_{B}$, namely

$$
N_{c B}(\tau)=\sum_{c \cdot \mu_{k}<\tau} 1=\sum_{\mu_{k}<\frac{\tau}{c}} 1=N_{B}\left(\frac{\tau}{c}\right) .
$$

In particular, (12) implies that, without loss of generality, we can assume ${ }^{1} \lambda_{j}>1$ and $\mu_{k}>1$ for all $j, k$. Let us now summarize the hypotheses on the factors $A_{1}, A_{2}$.

\section{Assumptions 1}

$M_{1}, M_{2}$ smooth closed manifolds of dimensions $n_{1}, n_{2}$, respectively;

$A=A_{1} \otimes A_{2}, A_{1} \in L_{\mathrm{cl}}^{1}\left(M_{1}\right), A_{2} \in L_{\mathrm{cl}}^{m_{2}}\left(M_{2}\right), \quad m_{2}>0, n_{1}>\frac{n_{2}}{m_{2}} ;$

$A_{1}, A_{2}$ positive, self-adjoint, elliptic;

$\sigma\left(A_{1}\right)=\left\{\lambda_{j}\right\}_{j \in \mathbb{N}}, \sigma\left(A_{2}\right)=\left\{\mu_{k}\right\}_{k \in \mathbb{N}}, \quad \lambda_{j}>1, \mu_{k}>1$, for all $j, k$.

Since $\lambda_{j}, \mu_{k}>1$ for all $j, k$, using (12), we have ${ }^{2}$

$$
\begin{aligned}
N_{A}(\tau) & =\sum_{\lambda_{j} \cdot \mu_{k}<\tau} 1=\sum_{\mu_{k}<\tau}\left(\sum_{\lambda_{j} \cdot \mu_{k}<\tau} 1\right) \\
& =\sum_{\mu_{k}<\tau} N_{\mu_{k} A_{1}}(\tau)=\sum_{\mu_{k}<\tau} N_{A_{1}}\left(\frac{\tau}{\mu_{k}}\right) .
\end{aligned}
$$

Proposition 3 Let $A, A_{1}$ and $A_{2}$ be as in Assumptions 1. Then,

$$
N_{A}(\tau)=\sum_{\mu_{k}<\tau}\left(\frac{C_{1}}{n_{1}}\left(\frac{\tau}{\mu_{k}}\right)^{n_{1}}+\frac{1}{\mu_{k}^{n_{1}-1}} r_{k}(\tau)\right),
$$

with

$$
C_{1}=\frac{1}{(2 \pi)^{n_{1}}} \int_{M_{1}} \int_{\mathbb{S}^{n_{1}-1}} \frac{d \theta_{1} d x_{1}}{\left[a_{m_{1}}\left(x_{1}, \theta_{1}\right)\right]^{\frac{n_{1}}{m_{1}}}},
$$

and $r_{k}(\tau)$ is $\mathcal{O}\left(\tau^{n_{1}-1}\right)$, uniformly with respect to $\mu_{k}$. That is, there exists a positive constant C such that

$$
\left|r_{k}(\tau)\right| \leq C \tau^{n_{1}-1}, \quad \text { for all } \quad k \in \mathbb{N} .
$$

\footnotetext{
${ }_{1}$ In fact, if that condition were not true, we could consider the operator $c^{2} A$, with $c=\left(\min \left\{\lambda_{j}, \mu_{k}\right\}-\varepsilon\right)^{-1}$, $\varepsilon>0$ small enough.

2 Recall that $\lambda_{j}>1$ for all $j$. In the first term of (13) we can reduce the summation to $\mu_{k}<\tau$ since, otherwise, we would have $\lambda_{k} \cdot \mu_{k} \geq \tau$ for all $k$, and the second summation would be zero.
} 
Proof By (13) we have

$$
N_{A}(\tau)=\sum_{\mu_{k}<\tau} N_{A_{1}}\left(\frac{\tau}{\mu_{k}}\right)
$$

Using (4), we can write

$$
N_{A}(\tau)=\sum_{\mu_{k}<\tau}\left(\frac{C_{1}}{n_{1}} \frac{\tau^{n_{1}}}{\mu_{k}^{n_{1}}}+R_{A}\left(\frac{\tau}{\mu_{k}}\right)\right) .
$$

Equation (5) implies that

$$
\left|R_{A}(t)\right| \leq \kappa t^{n_{1}-1}, \quad t>1,
$$

for a suitable constant $\kappa$. Since $\mu_{k}<\tau \Rightarrow \frac{\tau}{\mu_{k}}>1$ in the summation (16), we can write

$$
\left|R_{A}\left(\frac{\tau}{\mu_{k}}\right)\right| \leq C\left(\frac{\tau}{\mu_{k}}\right)^{n_{1}-1} .
$$

Hence, setting

$$
r_{k}(\tau)=\mu_{k}^{n_{1}-1} R_{A}\left(\frac{\tau}{\mu_{k}}\right)
$$

we have the assertion.

Lemma 1 Let $A, A_{1}, A_{2}$ be as in Assumptions 1, and assume $n_{1}>\frac{n_{2}}{m_{2}}$. Then, we have, for $\tau \rightarrow+\infty$,

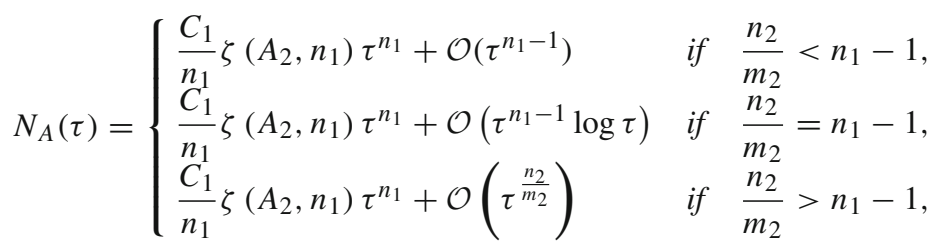

where $C_{1}$ is given by (14).

Proof Using Proposition 3, we obtain

$$
N_{A}(\tau)=\sum_{\mu_{k}<\tau}\left(\frac{C_{1}}{n_{1}}\left(\frac{\tau}{\mu_{k}}\right)^{n_{1}}+\frac{1}{\mu_{k}^{n_{1}-1}} r_{k}(\tau)\right),
$$

where $r_{k}(\tau)$ is uniformly $\mathcal{O}\left(\tau^{n_{1}-1}\right)$ for $\tau \rightarrow+\infty$, in the sense of (15). We can then write

$$
\begin{aligned}
\left|N_{A}(\tau)-\frac{C_{1}}{n_{1}} \zeta\left(A_{2}, n_{1}\right) \tau^{n_{1}}\right| & =\left|\sum_{\mu_{k}<\tau}\left(\frac{C_{1}}{n_{1}} \frac{\tau^{n_{1}}}{\mu_{k}^{n_{1}}}+\frac{1}{\mu_{k}^{n_{1}-1}} r_{k}\left(\tau^{n_{1}-1}\right)\right)-\frac{C_{1}}{n_{1}} \zeta\left(A_{2}, n_{1}\right) \tau^{n_{1}}\right| \\
& \leq \frac{C_{1}}{n_{1}} \tau^{n_{1}}\left|F_{A_{2}}\left(\tau, n_{1}\right)-\zeta\left(A_{2}, n_{1}\right)\right|+\left|\sum_{\mu_{k}<\tau} \frac{1}{\mu_{k}^{n_{1}-1}} r_{k}\left(\tau^{n_{1}-1}\right)\right| \\
& \leq \frac{C_{1}}{n_{1}} \tau^{n_{1}}\left|F_{A_{2}}\left(\tau, n_{1}\right)-\zeta\left(A_{2}, n_{1}\right)\right|+C \tau^{n_{1}-1} F_{A_{2}}\left(\tau, n_{1}-1\right) .
\end{aligned}
$$


Let us start with the case $n_{1}-1>\frac{n_{2}}{m}$. Using (17), we find

$$
\begin{aligned}
\limsup _{\tau \rightarrow+\infty} \frac{\left|N_{A}(\tau)-\frac{C_{1}}{n_{1}} \zeta\left(A_{2}, n_{1}\right) \tau^{n_{1}}\right|}{\tau^{n_{1}-1}} \leq & \frac{C_{1}}{n_{1}} \limsup _{\tau \rightarrow+\infty}\left|\zeta\left(A_{2}, n_{1}\right)-F_{A_{2}}\left(\tau, n_{1}\right)\right| \\
& +C \limsup _{\tau \rightarrow+\infty} F_{A_{2}}\left(\tau, n_{1}-1\right) .
\end{aligned}
$$

Since

$$
n_{1}>n_{1}-1>\frac{n_{2}}{m_{2}} \Rightarrow \frac{n_{2}}{m_{2}}-n_{1}<-1,
$$

$\zeta\left(A_{2}, n_{1}\right)-F_{1}(\tau)=\mathcal{O}\left(\tau^{\frac{n_{2}}{m_{2}}-n_{1}}\right)$ for $\tau \rightarrow+\infty$, in view of Proposition 1. It follows that

$$
\limsup _{\tau \rightarrow+\infty} \tau\left|\zeta\left(A_{2}, n_{1}\right)-F_{1}(\tau)\right| \leq \widetilde{C} \limsup _{\tau \rightarrow+\infty} \tau^{\frac{n_{2}}{m_{2}}-n_{1}+1}=0 \text {, }
$$

which implies

$$
\limsup _{\tau \rightarrow+\infty} \frac{\left|N_{A}(\tau)-\frac{C_{1}}{n_{1}} \zeta\left(A_{2}, n_{1}\right) \tau^{n_{1}}\right|}{\tau^{n_{1}-1}} \leq C \limsup _{\tau \rightarrow+\infty} F_{A_{2}}\left(\tau, n_{1}-1\right)=C \zeta\left(A_{2}, n_{1}-1\right) .
$$

Since $n_{1}-1>\frac{n_{2}}{m_{2}}, \zeta\left(A_{2}, n_{1}-1\right)$ is finite, and we have the desired assertion.

In the case $n_{1}-1=\frac{n_{2}}{m_{2}}$, from (17) we analogously get

$$
\begin{aligned}
& \limsup _{\tau \rightarrow+\infty} \frac{\left|N_{A}(\tau)-\frac{C_{1}}{n_{1}} \zeta\left(A_{2}, n_{1}\right) \tau^{n_{1}}\right|}{\tau^{n_{1}-1} \log \tau} \\
& \quad \leq \frac{C_{1}}{n_{1}} \limsup _{\tau \rightarrow+\infty} \frac{\tau}{\log \tau}\left|\zeta\left(A_{2}, n_{1}\right)-F_{A_{2}}\left(\tau, n_{1}\right)\right|+C \limsup _{\tau \rightarrow+\infty} \frac{1}{\log \tau} F_{A_{2}}\left(\tau, \frac{n_{2}}{m_{2}}\right) .
\end{aligned}
$$

Since $n_{1}>n_{1}-1=\frac{n_{2}}{m_{2}}$, in view of Proposition 1 we find

$$
\zeta\left(A_{2}, n_{1}\right)-F_{1}(\tau)=\mathcal{O}\left(\tau^{-1}\right), \quad F_{A_{2}}\left(\tau, \frac{n_{2}}{m_{2}}\right)=F_{2}(\tau)=\mathcal{O}(\log \tau),
$$

so that

$$
\limsup _{\tau \rightarrow+\infty} \frac{\left|N_{A}(\tau)-\frac{C_{1}}{n_{1}} \zeta\left(A_{2}, n_{1}\right) \tau^{n_{1}}\right|}{\tau^{n_{1}-1} \log \tau} \leq \widetilde{C}
$$

as claimed.

Finally, in the case $n_{1}-1<\frac{n_{2}}{m_{2}},(17)$ gives

$$
\begin{aligned}
\limsup _{\tau \rightarrow+\infty} \frac{\left|N_{A}(\tau)-\frac{C_{1}}{n_{1}} \zeta\left(A_{2}, n_{1}\right) \tau^{n_{1}}\right|}{\tau^{\frac{n_{2}}{m_{2}}}} \leq & \frac{C_{1}}{n_{1}} \limsup _{\tau \rightarrow+\infty} \tau^{n_{1}-\frac{n_{2}}{m_{2}}}\left|\zeta\left(A_{2}, n_{1}\right)-F_{A_{2}}\left(\tau, n_{1}\right)\right| \\
& +C \limsup _{\tau \rightarrow+\infty} \tau^{n_{1}-1-\frac{n_{2}}{m_{2}}} F_{A_{2}}\left(\tau, n_{1}-1\right) .
\end{aligned}
$$

Since $n_{1}>\frac{n_{2}}{m_{2}}>n_{1}-1$, Proposition 1 implies

$$
\zeta\left(A_{2}, n_{1}\right)-F_{1}(\tau)=\mathcal{O}\left(\tau^{\frac{n_{2}}{m_{2}}-n_{1}}\right), \quad F_{A_{2}}\left(\tau, n_{1}-1\right)=F_{3}(\tau)=\mathcal{O}\left(\tau^{\frac{n_{2}}{m}-n_{1}+1}\right) .
$$


Therefore,

$$
\limsup _{\tau \rightarrow+\infty} \frac{\left|N_{A}(\tau)-\frac{C_{1}}{n_{1}} \zeta\left(A_{2}, n_{1}\right) \tau^{n_{1}}\right|}{\tau^{\frac{n_{2}}{m_{2}}}}<+\infty .
$$

The proof is complete.

We can now prove our main result.

Theorem 2 Let $M_{1}, M_{2}$ be two closed manifolds of dimension $n_{1}, n_{2}$, respectively. Let $A=A_{1} \otimes A_{2}$, where $A_{j} \in L_{\mathrm{cl}}^{m_{j}}\left(M_{j}\right), m_{j}>0, j=1,2$, are positive, self-adjoint, invertible operators, with $\frac{n_{1}}{m_{1}}>\frac{n_{2}}{m_{2}}$. Then, for $\tau \rightarrow+\infty$,

$$
N_{A}(\tau)= \begin{cases}\frac{C_{1}}{n_{1}} \zeta\left(A_{2}, \frac{n_{1}}{m_{1}}\right) \tau^{\frac{n_{1}}{m_{1}}}+\mathcal{O}\left(\tau^{\frac{n_{1}-1}{m_{1}}}\right) & \text { if } \frac{n_{2}}{m_{2}}<\frac{n_{1}-1}{m_{1}}, \\ \frac{C_{1}}{n_{1}} \zeta\left(A_{2}, \frac{n_{1}}{m_{1}}\right) \tau^{\frac{n_{1}}{m_{1}}}+\mathcal{O}\left(\tau^{\frac{n_{1}-1}{m_{1}}} \log \tau\right) & \text { if } \frac{n_{2}}{m_{2}}=\frac{n_{1}-1}{m_{1}}, \\ \frac{C_{1}}{n_{1}} \zeta\left(A_{2}, \frac{n_{1}}{m_{1}}\right) \tau^{\frac{n_{1}}{m_{1}}}+\mathcal{O}\left(\tau^{\frac{n_{2}}{m_{2}}}\right) & \text { if } \frac{n_{2}}{m_{2}}>\frac{n_{1}-1}{m_{1}},\end{cases}
$$

where $C_{1}$ is given by (14).

Proof Without loss of generality, we can assume $m_{1}=1$, possibly considering an appropriate power of $A$, see [4]. Moreover, again without loss of the generality, we can assume that all the eigenvalues are strictly larger than one, so that the Assumptions 1 are fulfilled. Then, the claim follows from Lemma 1.

The case of the tensor product of two Shubin operators can be treated in a completely similar fashion, using Proposition 2 in place of Proposition 1, and the Weyl law (6) which holds in this setting.

Theorem 3 Let $P=P_{1} \otimes P_{2}$ and $P_{j} \in G_{\mathrm{cl}}^{m_{j}}\left(\mathbb{R}^{n_{j}}\right), m_{j}>0, j=1,2$, be positive, selfadjoint, invertible operators, with $\frac{2 n_{1}}{m_{1}}>\frac{2 n_{2}}{m_{2}}$. Then, for $\tau \rightarrow+\infty$,

$$
N_{P}(\tau)= \begin{cases}\frac{K_{1}}{2 n_{1}} \zeta\left(P_{2}, \frac{2 n_{1}}{m_{1}}\right) \tau^{\frac{2 n_{1}}{m_{1}}}+\mathcal{O}\left(\tau^{\frac{2 n_{1}-1}{m_{1}}}\right) & \text { if } \frac{2 n_{2}}{m_{2}}<\frac{2 n_{1}-1}{m_{1}}, \\ \frac{K_{1}}{2 n_{1}} \zeta\left(P_{2}, \frac{2 n_{1}}{m_{1}}\right) \tau^{\frac{2 n_{1}}{m_{1}}}+\mathcal{O}\left(\tau^{\frac{2 n_{1}-1}{m_{1}}} \log \tau\right) & \text { if } \frac{2 n_{2}}{m_{2}}=\frac{2 n_{1}-1}{m_{1}} \\ \frac{K_{1}}{2 n_{1}} \zeta\left(P_{2}, \frac{2 n_{1}}{m_{1}}\right) \tau^{\frac{2 n_{1}}{m_{1}}}+\mathcal{O}\left(\tau^{\frac{2 n_{2}}{m_{2}}}\right) & \text { if } \frac{2 n_{2}}{m_{2}}>\frac{2 n_{1}-1}{m_{1}}\end{cases}
$$

where

$$
K_{1}=\frac{1}{(2 \pi)^{2 n_{1}}} \int_{\mathbb{S}^{2 n_{1}-1}} \frac{d \theta_{1}}{\left[p_{m_{1}}\left(\theta_{1}\right)\right]^{\frac{2 n_{1}}{m_{1}}}} .
$$

\section{Spectral asymptotics for the tensor product of $r$ operators}

As in the previous sections, to avoid redundancy we will prove in detail our results for tensor products of $r$ factors only in the case of operators belonging to the Hörmander calculus on 
closed manifolds. We will then omit the proof of the analogous Theorem 4 for the case of operators belonging to the Shubin calculus, which can be obtained by similar arguments.

The main tool in the study of the extension of Theorem 2 to the product of $r \geq 2$ factors is a refined version of Proposition 1. Let us first state the hypotheses.

\section{Assumptions 2}

$M_{1}, \ldots, M_{r}$ smooth closed manifolds of dimensions $n_{1}, \ldots, n_{r}$, respectively;

$A=A_{1} \otimes \cdots \otimes A_{r}, \quad A_{j} \in L_{\mathrm{cl}}^{m_{j}}\left(M_{j}\right), m_{j}>0, \quad j=1, \ldots, r ;$

$A_{j}$ positive, self-adjoint, elliptic $, \quad j=1, \ldots, r$;

$\sigma\left(A_{j}\right)=\left\{{ }^{j} \mu_{k_{j}}\right\}_{k_{j} \in \mathbb{N}}, \quad{ }^{j} \mu_{1}>1, \quad j=1, \ldots, r$.

Proposition 4 Let $A, A_{j}, j=1, \ldots, r$, be as in Assumptions 2. Set

$$
p=\max \left\{\frac{n_{1}}{m_{1}}, \ldots, \frac{n_{r}}{m_{r}}\right\}, \quad S=\left\{j \in\{1, \ldots, r\}: \frac{n_{j}}{m_{j}}=p\right\}, \quad s=\sharp S,
$$

and define, for $\tau \rightarrow+\infty$,

$$
F_{A}(\tau, c)=\sum_{{ }_{1} \mu_{k_{1}} \cdot \ldots \cdot{ }^{r} r_{k_{r}}<\tau} \frac{1}{\left({ }^{1} \mu_{k_{1}}\right)^{c} \cdot \ldots \cdot\left({ }^{r} \mu_{k_{r}}\right)^{c}}= \begin{cases}F_{1}(\tau) & \text { if } p<c, \\ F_{2}(\tau) & \text { if } p=c, \\ F_{3}(\tau) & \text { if } p>c .\end{cases}
$$

Then,

$$
\begin{array}{r}
\limsup _{\tau \rightarrow+\infty} \frac{\left|\prod_{j=1}^{r} \zeta\left(A_{j}, c\right)-F_{1}(\tau)\right|}{\tau^{p-c}(\log \tau)^{s-1}}=\kappa_{1}, \\
\limsup _{\tau \rightarrow+\infty} \frac{F_{2}(\tau)}{(\log \tau)^{s}}=\kappa_{2}, \\
\limsup _{\tau \rightarrow+\infty} \frac{F_{3}(\tau)}{\tau^{p-c}(\log \tau)^{s-1}}=\kappa_{3},
\end{array}
$$

that is, for $\tau \rightarrow+\infty$,

$$
\begin{aligned}
\prod_{j=1}^{r} \zeta\left(A_{j}, c\right)-F_{1}(\tau) & =\mathcal{O}\left(\tau^{p-c}(\log \tau)^{s-1}\right), \\
F_{2}(\tau) & =\mathcal{O}\left((\log \tau)^{s}\right), F_{3}(\tau)=\mathcal{O}\left(\tau^{p-c}(\log \tau)^{s-1}\right) .
\end{aligned}
$$

Proof We will make use of the straightforward inequality

$$
F_{A}(\tau, c)=\sum_{{ }^{1} \mu_{k_{1}} \cdot \ldots \cdot{ }^{r} \mu_{k_{r}<\tau}} \frac{1}{\left({ }^{1} \mu_{k_{1}}\right)^{c} \cdot \ldots \cdot\left({ }^{r} \mu_{k_{r}}\right)^{c}} \leq \prod_{j=1}^{r} \sum_{j} \frac{1}{\mu_{\mu_{k_{j}}<\tau}} \frac{1}{\left({ }^{j} \mu_{k_{j}}\right)^{c}},
$$

as well as of the following consequence of the absolute convergence of the involved series,

$$
\prod_{j=1}^{r} \zeta\left(A_{j}, c\right)=\lim _{\tau \rightarrow+\infty} \prod_{j=1}^{r} \sum_{j} \frac{1}{\mu_{\mu_{j}}<\tau} \frac{1}{\left({ }^{j} \mu_{k_{j}}\right)^{c}}=\sum \frac{1}{\left({ }^{1} \mu_{k_{1}}\right)^{c} \cdot \ldots \cdot\left({ }^{r} \mu_{k_{r}}\right)^{c}},
$$


where $c$ belongs to the holomorphic domain of the functions $\zeta\left(A_{j}, z\right), j=1, \ldots, r$, and the last summation in (19) is taken on all the $r$-tuples of eigenvalues $\left({ }^{1} \mu_{k_{1}}, \ldots,{ }^{r} \mu_{k_{r}}\right) \in$ $\sigma\left(A_{1}\right) \oplus \cdots \oplus \sigma\left(A_{r}\right)$.

Case $p=c$. Let us split the last term in (18) as

$$
\prod_{j=1}^{r} \sum_{j} \frac{1}{{ }_{\mu_{k_{j}}<\tau}} \frac{1}{\left({ }^{j} \mu_{k_{j}}\right)^{c}}=\left(\prod_{j \notin S} \sum_{j} \frac{1}{{ }^{\prime} \mu_{k_{j}}<\tau} \frac{1}{\left({ }^{j} \mu_{k_{j}}\right)^{c}}\right) \cdot\left(\prod_{t \in S} \sum_{t} \frac{1}{{ }^{t} \mu_{k_{t}<\tau}} \frac{1}{\left({ }^{t} \mu_{k_{t}}\right)^{c}}\right) .
$$

Recalling that $\frac{n_{j}}{m_{j}}<c$ for all $j \notin S$, that is, $c$ belongs to the holomorphic domain of $\zeta\left(A_{j}, \cdot\right)$ for $j \notin S$, and that $\frac{n_{t}}{m_{t}}=c$ for all $t \in S$, using Proposition 1 we have

$$
\begin{aligned}
& \left(\prod_{j \notin S} \sum_{j} \frac{1}{{ }_{j} \mu_{k_{j}<\tau}} \frac{1}{\left({ }^{j} \mu_{k_{j}}\right)^{c}}\right) \cdot\left(\prod_{t \in S} \sum_{t} \frac{1}{{ }^{\mu_{k_{t}}<\tau}} \frac{1}{\left.{ }^{t} \mu_{k_{t}}\right)^{c}}\right)=\left(\prod_{j \notin S} \zeta\left(A_{j}, c\right)\right) \cdot \mathcal{O}\left((\log \tau)^{S}\right) \\
& =\mathcal{O}\left((\log \tau)^{S}\right),
\end{aligned}
$$

which implies our claim in this case, in view of (18).

Case $p>c$. To simplify notation, we can suppose, without loss of generality, $p=\frac{n_{1}}{m_{1}}$. Recalling the assumption ${ }^{j} \mu_{k_{j}}>1, j=1, \ldots, r$, we observe that

$$
{ }^{1} \mu_{k_{1}} \cdot \ldots \cdot{ }^{r} \mu_{k_{r}}<\tau \Leftrightarrow\left[\prod_{j=2}^{r}\left({ }^{j} \mu_{k_{j}}\right)<\tau \wedge 1<{ }^{1} \mu_{k_{1}}<\frac{\tau}{\prod_{j=2}^{r}\left({ }^{j} \mu_{k_{j}}\right)}\right] .
$$

In fact, the $\Leftarrow$ implication is immediate, while

$$
\begin{aligned}
& { }^{1} \mu_{k_{1}} \cdot \ldots{ }^{r} \mu_{k_{r}}<\tau \wedge{ }^{1} \mu_{k_{1}}>1 \\
& \Rightarrow 1<{ }^{1} \mu_{k_{1}}<\frac{\tau}{\left.\prod_{j=2}^{r}{ }^{j} \mu_{k_{j}}\right)} \\
& \Rightarrow 1<{ }^{1} \mu_{k_{1}}<\frac{\tau}{\prod_{j=2}^{r}\left({ }^{j} \mu_{k_{j}}\right)} \wedge \prod_{j=2}^{r}\left({ }^{j} \mu_{k_{j}}\right)<\tau .
\end{aligned}
$$

Then, we can write

$$
\begin{aligned}
& F_{A}(\tau, c)=F_{3}(\tau) \\
& =\sum_{{ }^{2} \mu_{k_{2}} \cdot \ldots \cdot{ }^{r} \mu_{k_{r}}<\tau} \frac{1}{\left({ }^{2} \mu_{k_{2}}\right)^{c} \cdot \ldots \cdot\left({ }^{r} \mu_{k_{r}}\right)^{c}} \sum_{1<1} \sum_{\mu_{k_{1}}<\frac{\tau}{{ }^{2} \mu_{k_{2}} \ldots \cdot{ }^{r} \mu_{k_{r}}}} \frac{1}{\left({ }^{1} \mu_{k_{1}}\right)^{c}} \\
& =\sum_{\prod_{j=2}^{r}\left({ }^{j} \mu_{k_{j}}\right)<\tau} \frac{1}{\prod_{j=2}^{r}\left({ }^{j} \mu_{k_{j}}\right)^{c}} \int_{1}^{\frac{\tau}{\prod_{\left.j=2^{(j} \mu_{k_{j}}\right)}^{r}}} \frac{1}{\mu^{c}} \mathrm{~d} N_{A_{1}}(\mu) .
\end{aligned}
$$


Switching to $B=\left(A_{1}\right)^{\frac{1}{m_{1}}}$, recalling that then $\sigma(B)=\left\{\left({ }^{1} \mu_{k_{1}}\right)^{\frac{1}{m_{1}}}\right\}$ and $^{3}$

$$
\int_{1}^{\frac{\tau}{\prod_{\left.j=2^{(j} \mu_{k_{j}}\right)}^{r}}} \frac{1}{\mu^{c}} \mathrm{~d} N_{A_{1}}(\mu)=\int_{1}^{[}\left[\frac{\tau}{\prod_{j=2^{r}}^{\left.r^{j} \mu_{k_{j}}\right)}}\right]^{\frac{1}{m_{1}}} \frac{1}{\mu^{c m_{1}}} \mathrm{~d} N_{B}(\mu),
$$

using (9) with $n_{1}$ in place of $n$, it turns out that, for $\tau \rightarrow+\infty$,

$$
\begin{aligned}
F_{3}(\tau) & =\sum_{\prod_{j=2}^{r}\left({ }^{j} \mu_{k_{j}}\right)<\tau} \frac{1}{\prod_{j=2}^{r}\left({ }^{j} \mu_{k_{j}}\right)^{c}} \mathcal{O}\left(\left(\frac{\tau}{\prod_{j=2}^{r}\left({ }^{j} \mu_{k_{j}}\right)}\right)^{p-c}\right) \\
& =\left[\sum_{{ }^{2} \mu_{k_{2}} \cdot{ }^{r}{ }^{r} \mu_{k_{r}<\tau}} \frac{1}{\left({ }^{2} \mu_{k_{2}}\right)^{p} \cdot \ldots \cdot\left({ }^{r} \mu_{k_{r}}\right)^{p}}\right] \mathcal{O}\left(\tau^{p-c}\right) .
\end{aligned}
$$

Using the result of the case $p=c$ above, with $s-1$ in place of $s$, we conclude

$$
F_{3}(\tau)=\mathcal{O}\left(\tau^{p-c}(\log \tau)^{s-1}\right),
$$

as claimed.

Case $p<c$. Since $c>\frac{n_{j}}{m_{j}}$ for all $j=1, \ldots, r, c$ belongs to the holomorphic domain of all the functions $\zeta\left(A_{j}, z\right), j=1, \ldots, r$. Then, by (19), in this case we have, for all $\tau$,

$$
\begin{aligned}
& \prod_{j=1}^{r} \zeta\left(A_{j}, c\right)-F_{A}(\tau, c)=\prod_{j=1}^{r} \zeta\left(A_{j}, c\right)-F_{1}(\tau) \\
& =\sum \frac{1}{\left({ }^{1} \mu_{k_{1}}\right)^{c} \cdot \ldots \cdot\left({ }^{r} \mu_{k_{r}}\right)^{c}}-\sum_{{ }^{1} \mu_{k_{1}} \cdot \ldots \cdot{ }^{r} \mu_{k_{r}<\tau}} \frac{1}{\left({ }^{1} \mu_{k_{1}}\right)^{c} \cdot \ldots \cdot\left({ }^{r} \mu_{k_{r}}\right)^{c}} \\
& =\sum_{{ }^{1} \mu_{k_{1}} \cdot \ldots \cdot{ }^{r} \mu_{k_{r} \geq \tau}} \frac{1}{\left({ }^{1} \mu_{k_{1}}\right)^{c} \cdot \ldots \cdot\left({ }^{r} \mu_{k_{r}}\right)^{c}} .
\end{aligned}
$$

We will prove the claim by induction on the number of operators. The case $r=2$ is proven in Proposition 1. Let us then suppose that the desired estimate holds true for a tensor product of $r-1$ operators, $r \geq 2$, and let us prove that it holds true also for a tensor product of $r$ operators.

We can again suppose, without loss of generality, $p=\frac{n_{1}}{m_{1}}$. Since, clearly,

$$
\begin{aligned}
& { }^{1} \mu_{k_{1}} \cdot \ldots \cdot{ }^{r} \mu_{k_{r}} \geq \tau \Rightarrow \\
& { }^{1} \mu_{k_{1}} \geq \frac{\tau}{\prod_{j=2}^{r}\left({ }^{j} \mu_{k_{j}}\right)} \wedge\left[\prod_{j=2}^{r}\left({ }^{j} \mu_{k_{j}}\right)<\tau \vee \prod_{j=2}^{r}\left({ }^{j} \mu_{k_{j}}\right) \geq \tau\right],
\end{aligned}
$$

3 That is,

$$
\sum_{1<{ }^{1} \mu_{k_{1}}<\frac{\tau}{{ }^{2} \mu_{k_{2}} \ldots \cdot{ }^{r} \mu_{k_{r}}}}=\frac{1}{\left({ }^{1} \mu_{k_{1}}\right)^{c}}=\sum_{1<\left({ }^{1} \mu_{k_{1}}\right)^{\frac{1}{m_{1}}}<\left[\frac{\tau}{{ }^{2} \mu_{k_{2}} \cdot \ldots \cdot{ }^{r} \mu_{k_{r}}}\right]^{\frac{1}{m_{1}}}}=\frac{1}{\left[\left({ }^{1} \mu_{k_{1}}\right)^{\frac{1}{m_{1}}}\right]^{c m_{1}}}
$$

similar to the proof of Proposition 1. 
we can write

$$
\begin{aligned}
& \prod_{j=1}^{r} \zeta\left(A_{j}, c\right)-F_{1}(\tau) \\
& =\sum_{\prod_{j=2}^{r}\left({ }^{j} \mu_{k_{j}}\right)<\tau} \frac{1}{\prod_{j=2}^{r}\left({ }^{j} \mu_{k_{j}}\right)^{c}} \int_{\frac{\tau}{\prod_{\left.j=2^{(j} \mu_{k_{j}}\right)}^{+\infty}} \frac{1}{\mu^{c}} d N_{A_{1}}(\mu)}^{+\infty} \frac{1}{\quad+\sum_{\prod_{j=2}^{r}\left({ }^{j} \mu_{k_{j}}\right) \geq \tau} \frac{1}{\prod_{j=2}^{r}\left({ }^{j} \mu_{k_{j}}\right)^{c}} \int_{\frac{\tau}{\left.\prod_{j=2}^{r}{ }^{(j} \mu_{k_{j}}\right)}}^{\mu^{c}} d N_{A_{1}}(\mu) .}
\end{aligned}
$$

Let us first consider (20). Arguing as in the previous case $p>c$, and using the case $p=c$ with $s-1$ in place of $s$, we find, for $\tau \rightarrow+\infty$,

$$
\begin{aligned}
& \sum_{\prod_{j=2}^{r}\left({ }^{j} \mu_{k_{j}}\right)<\tau} \frac{1}{\prod_{j=2}^{r}\left(^{j} \mu_{k_{j}}\right)^{c}} \int_{\frac{\tau}{\prod_{\left.j=2^{(j} \mu_{k_{j}}\right)}^{r}}}^{+\infty} \frac{1}{\mu^{c}} d N_{A_{1}}(\mu) \\
= & \sum_{\prod_{j=2}^{r}\left({ }^{j} \mu_{k_{j}}\right)<\tau} \frac{1}{\prod_{j=2}^{r}\left({ }^{j} \mu_{k_{j}}\right)^{c}} \mathcal{O}\left(\left(\frac{\tau}{\prod_{j=2}^{r}{ }^{j} \mu_{k_{j}}}\right)^{p-c}\right) \\
= & \mathcal{O}\left(\tau^{p-c}(\log \tau)^{s-1}\right),
\end{aligned}
$$

which is the desired estimate. We now show that (21) fulfills the same estimate. Using the fact that $\zeta\left(A_{1}, c\right)$ is finite, we can estimate (21) as

$$
\sum_{\prod_{j=2}^{r}\left({ }^{j} \mu_{k_{j}}\right) \geq \tau} \frac{1}{\prod_{j=2}^{r}\left({ }^{j} \mu_{k_{j}}\right)^{c}} \int_{\frac{\tau}{\prod_{j=2}^{r}{ }^{\left(j_{\mu_{k}}\right)}}}^{+\infty} \frac{1}{\mu^{c}} d N_{A_{1}}(\mu) \leq \sum_{\left.\prod_{j=2}^{r}{ }^{j} \mu_{k_{j}}\right) \geq \tau} \frac{1}{\left.\prod_{j=2}^{r}{ }^{j} \mu_{k_{j}}\right)^{c}} \zeta\left(A_{1}, c\right) .
$$

By the inductive hypothesis, we see that (22) is $\mathcal{O}\left(\tau^{\tilde{p}-c}(\log \tau)^{\widetilde{s}-1}\right)$ for $\tau \rightarrow+\infty$, where $\tilde{p}=\max \left\{\frac{n_{j}}{m_{j}}\right\}_{j=2}^{r} \leq p$ and $\widetilde{s}<s$. Therefore, it is also $\mathcal{O}\left(\tau^{p-c}(\log \tau)^{s-1}\right)$ for $\tau \rightarrow+\infty$, and the same of course holds for (21), in view of the above estimate.

The proof is complete.

Assumptions 3 Let $A, A_{1}, \ldots, A_{r}$ be as in Assumptions 2, and suppose that there exists $l \in\{1, \ldots, r\}$ such that

$$
\frac{n_{l}}{m_{l}}>\max \left\{\frac{n_{j}}{m_{j}}\right\}_{j \in\{1, \ldots, r\} \backslash\{l\}} .
$$

For notational simplicity, in the next two statements we also assume, without loss of generality, that $l=1$. As in the previous section, we first consider the case when $m_{1}=1$. We will denote by $\mu_{\mathbf{j}_{\geq 2}}$ the product $\prod_{j=2}^{r}{ }^{j} \mu_{k_{j}}$, where $\mathbf{j}_{\geq 2}$ denotes the multi-index $\left(k_{2}, \ldots, k_{r}\right) \in$ $\mathbb{N}^{r-1}$. The following proposition is an extension of Proposition 5 .

Proposition 5 Let $A, A_{1}, \ldots, A_{r}$ be as in Assumptions 3. Then,

$$
N_{A}(\tau)=\sum_{\mu_{\mathbf{j} \geq 2}<\tau}\left(\frac{C_{1}}{n_{1}}\left(\frac{\tau}{\mu_{\mathbf{j}_{\geq 2}}}\right)^{n_{1}}+\frac{1}{\mu_{\mathbf{j}_{\geq 2}}^{n_{1}-1}} r_{\mathbf{j} \geq 2}(\tau)\right),
$$


where $C_{1}$ is given by (14) and $r_{\mathbf{j} \geq 2}$ is $\mathcal{O}\left(\tau^{n_{1}-1}\right)$, uniformly with respect to $\mu_{\mathbf{j} \geq 2}$, for any $\mathbf{j}_{\geq 2}$. That is, there exists a positive constant $C$ such that

$$
\left|r_{\mathbf{j} \geq 2}(\tau)\right| \leq C \tau^{n_{1}-1}, \quad \text { for all } \quad \mathbf{j}_{\geq 2} \in \mathbb{N}^{r-1} .
$$

Proposition 5 implies the next lemma, which is a multidimensional version of Lemma 1. We omit the proof, since the argument is analogue to the one used to prove Lemma 1, similar to what has been done in the proof of Proposition 4.

Lemma 2 Let $A, A_{1}, \ldots, A_{r}$ be as in Assumptions 3. Let us suppose that $m_{1}=1$ and $n_{1}>\frac{n_{j}}{m_{j}}, j=2, \ldots, r$ and $s e t$

$$
p=\max \left\{\frac{n_{j}}{m_{j}}\right\}_{j=2, \ldots, r}, \quad S=\left\{j=2, \ldots, r: \frac{n_{j}}{m_{j}}=p\right\}, \quad s=\sharp S .
$$

Then, we have, for $\tau \rightarrow+\infty$,

$$
N_{A}(\tau)= \begin{cases}C_{A} \tau^{n_{1}}+\mathcal{O}\left(\tau^{n_{1}-1}\right) & \text { if } p<n_{1}-1 \\ C_{A} \tau^{n_{1}}+\mathcal{O}\left(\tau^{n_{1}-1}(\log \tau)^{s}\right) & \text { if } p=n_{1}-1 \\ C_{A} \tau^{n_{1}}+\mathcal{O}\left(\tau^{p}(\log \tau)^{s-1}\right) & \text { if } p>n_{1}-1\end{cases}
$$

where

$$
C_{A}=\frac{C_{1}}{n_{1}} \prod_{j=2}^{r} \zeta\left(A_{j}, n_{1}\right)
$$

and $C_{1}$ is given by (14).

Finally, using powers of the operator $A$, it is possible to extend the result to the case where all the factors have arbitrary positive order, which is, together with Theorem 5 below for the tensor product of $r$ factors in the Shubin calculus, our next main result.

Theorem 4 Let $M_{1}, \ldots, M_{r}$ be closed manifolds of dimension $n_{1}, \ldots, n_{r}$, respectively. Let $A=A_{1} \otimes \cdots \otimes A_{r}$, where $A_{j} \in L_{\mathrm{cl}}^{m_{j}}\left(M_{j}\right), m_{j}>0, j=1, \ldots, r$, are positive, selfadjoint, invertible operators, and assume that there exists $l \in\{1, \ldots, r\}$ such that $\frac{n_{l}}{m_{l}}>$ $\max \left\{\frac{n_{j}}{m_{j}}\right\}_{j \in\{1, \ldots, r\} \backslash\{l\}}$. Set

$$
p=\max \left\{\frac{n_{j}}{m_{j}}\right\}_{j \in\{1, \ldots, r\} \backslash\{l\}}, S=\left\{j=1, \ldots, r, j \neq l: \frac{n_{j}}{m_{j}}=p\right\}, s=\sharp S .
$$

Then, for $\tau \rightarrow+\infty$,

$$
N_{A}(\tau)=\left\{\begin{array}{lll}
C_{A} \tau^{\frac{n_{l}}{m_{l}}}+\mathcal{O}\left(\tau^{\frac{n_{l}-1}{m_{l}}}\right) & \text { if } & p<\frac{n_{l}-1}{m_{l}}, \\
C_{A} \tau^{\frac{n_{l}}{m_{l}}}+\mathcal{O}\left(\tau^{\frac{n_{l}-1}{m_{l}}}(\log \tau)^{s}\right) & \text { if } & p=\frac{n_{l}-1}{m_{l}}, \\
C_{A} \tau^{\frac{n_{l}}{m_{l}}}+\mathcal{O}\left(\tau^{p}(\log \tau)^{s-1}\right) & \text { if } & p>\frac{n_{l}-1}{m_{l}},
\end{array}\right.
$$

where

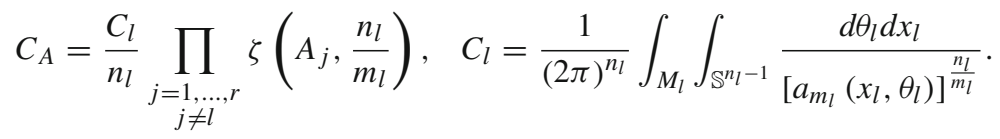


Theorem 5 Let $P=P_{1} \otimes \cdots \otimes P_{r}$ and $P_{j} \in G_{\mathrm{cl}}^{m_{j}}\left(\mathbb{R}^{n_{j}}\right), m_{j}>0, j=1, \ldots, r$, be positive, self-adjoint, invertible operators, and assume that there exists $l \in\{1, \ldots, r\}$ such that $\frac{2 n_{l}}{m_{l}}>\max \left\{\frac{2 n_{j}}{m_{j}}\right\}_{j \in\{1, \ldots, r\} \backslash\{l\}}$. Set

$$
p=\max \left\{\frac{2 n_{j}}{m_{j}}\right\}_{j \in\{1, \ldots, r\} \backslash\{l\}}, S=\left\{j=1, \ldots, r, j \neq l: \frac{2 n_{j}}{m_{j}}=p\right\}, s=\sharp S .
$$

Then, for $\tau \rightarrow+\infty$,

$$
N_{P}(\tau)= \begin{cases}K_{P} \tau^{\frac{2 n_{l}}{m_{l}}}+\mathcal{O}\left(\tau^{\frac{2 n_{l}-1}{m_{l}}}\right) & \text { if } p<\frac{2 n_{l}-1}{m_{l}}, \\ K_{P} \tau^{\frac{2 n_{l}}{m_{l}}}+\mathcal{O}\left(\tau^{\frac{2 n_{l}-1}{m_{l}}}(\log \tau)^{s}\right) & \text { if } p=\frac{2 n_{l}-1}{m_{l}}, \\ K_{P} \tau^{\frac{2 n_{l}}{m_{l}}}+\mathcal{O}\left(\tau^{p}(\log \tau)^{s-1}\right) & \text { if } p>\frac{2 n_{l}-1}{m_{l}},\end{cases}
$$

where

$$
K_{P}=\frac{V_{l}}{2 n_{l}} \prod_{\substack{j=1, \ldots, r \\ j \neq l}} \zeta\left(P_{j}, \frac{2 n_{l}}{m_{l}}\right), \quad V_{l}=\frac{1}{(2 \pi)^{2 n_{l}}} \int_{\mathbb{S}^{2 n-1}} \frac{d \theta_{l}}{\left[p_{m_{l}}\left(\theta_{l}\right)\right]^{\frac{2 n_{l}}{m_{l}}}} .
$$

\section{Sharpness of the result}

In this section, we show that the estimates obtained in Theorem 2 are sharp. To begin, we choose two pseudodifferential operators on spheres, whose spectrum we can describe explicitly. Namely, we set

$$
A_{1}=\left(-\Delta_{\mathbb{S}^{2}}+2\right)-2\left(-\Delta_{\mathbb{S}^{2}}+\frac{1}{4}\right)^{\frac{1}{2}} \in L_{\mathrm{cl}}^{2}\left(\mathbb{S}^{2}\right), A_{2}=-\Delta_{\mathbb{S}^{1}}+1 \in L_{\mathrm{cl}}^{2}\left(\mathbb{S}^{1}\right),
$$

where $A_{1}$ is considered as an unbounded operator on $L^{2}\left(\mathbb{S}^{2}\right)$, where $\mathbb{S}^{2}$ is the two-dimensional sphere, and $A_{2}$ is considered as an unbounded operator on $L^{2}\left(\mathbb{S}^{1}\right)$, where $\mathbb{S}^{1}$ is the onedimensional sphere. It is well known, see, e.g., [26, §3], that

$$
\begin{aligned}
& \sigma\left(-\Delta_{\mathbb{S}^{2}}\right)=\left\{k^{2}+k \mid k \in \mathbb{N}, \text { mult }\left(k^{2}+k\right)=(2 k+1)\right\}, \\
& \sigma\left(-\Delta_{\mathbb{S}^{1}}\right)=\left\{n^{2} \mid n \in \mathbb{N}, \text { mult }\left(n^{2}\right)=2\right\},
\end{aligned}
$$

where mult $(\tau)$ is the multiplicity of the eigenvalue $\tau$. Therefore, by the functional calculus of operators,

$$
\begin{aligned}
& \sigma\left(A_{1}\right)=\left\{k^{2}-k+1 \mid k \in \mathbb{N}, \text { mult }\left(k^{2}-k+1\right)=(2 k+1)\right\}, \\
& \sigma\left(A_{2}\right)=\left\{n^{2}+1 \mid n \in \mathbb{N}, \text { mult }\left(n^{2}+1\right)=2\right\},
\end{aligned}
$$

since the eigenfunction of $A_{1}$ and $-\Delta_{\mathbb{S}^{2}}$ are the same. Notice that all the eigenvalues of $A_{1}$ are larger then 1; therefore,

$$
N_{A_{1}}(\tau)=0, \quad \tau \leq 1
$$


Knowing precisely the eigenvalues of $A_{1}$ together with their multiplicities, we can write, for $\tau>1$,

$$
\begin{aligned}
N_{A_{1}}(\tau) & =\sum_{k^{2}-k+1<\tau} \operatorname{mult}\left(k^{2}-k+1\right) \\
& =\sum_{k^{2}-k+1<\tau}(2 k+1)=\sum_{k=0}^{k}(2 k+1)
\end{aligned}
$$

where

$$
\bar{k}^{2}-\bar{k}+1<\tau \leq(\bar{k}+1)^{2}-(\bar{k}+1)+1=\bar{k}^{2}+\bar{k}+1, \tau>1 .
$$

That is,

$$
N_{A_{1}}(\tau)=\sum_{k=0}^{\bar{k}}(2 k+1)=\sum_{k^{2}+k \leq \bar{k}^{2}+\bar{k}} \text { mult }\left(k^{2}+k\right)=N_{-\Delta_{\mathbb{S}^{2}}}\left(\bar{k}^{2}+\bar{k}+\frac{1}{2}\right),
$$

provided that

$$
\bar{k}^{2}-\bar{k}+1<\tau \leq(\bar{k}+1)^{2}-(\bar{k}+1)+1=\bar{k}^{2}+\bar{k}+1, \tau>1 .
$$

Using a well-known result on the counting function of the Laplacian on the spheres (see [26]), we have, for each $\bar{k} \in \mathbb{N}$,

$$
N_{-\Delta_{\mathbb{S}^{2}}}\left(\bar{k}^{2}+\bar{k}+\frac{1}{2}\right)=\bar{k}^{2}+2 \bar{k}+1 .
$$

So, in view of (26), supposing $\tau>1$, we find

$$
\begin{aligned}
N_{A_{1}}(\tau)= & \bar{k}^{2}+2 \bar{k}+1, \\
& \bar{k}^{2}-\bar{k}+1<\tau \leq(\bar{k}+1)^{2}-(\bar{k}+1)+1=\bar{k}^{2}+\bar{k}+1 .
\end{aligned}
$$

The asymptotic expansion (4) implies that

$$
N_{A_{1}}(\tau)=\tau+R(\tau), \quad R=\mathcal{O}\left(\tau^{\frac{1}{2}}\right) .
$$

We can then obtain a bound for $R(\tau)$ :

$$
\begin{aligned}
R(\tau) & =N_{A_{1}}(\tau)-\tau \\
& =\bar{k}^{2}+2 \bar{k}+1-\tau, \quad \bar{k}^{2}-\bar{k}+1<\tau \leq \bar{k}^{2}+\bar{k}+1 .
\end{aligned}
$$

Therefore, for $\tau>16$,

$$
R(\tau) \geq \bar{k}^{2}+2 \bar{k}+1-\bar{k}^{2}-\bar{k}-1=\bar{k}>\frac{3 \sqrt{\tau}}{4},
$$

which implies, in particular, that the remainder is positive for $\tau>16$. We also have

$$
R(\tau)<\bar{k}^{2}+2 \bar{k}+1-\bar{k}^{2}+\bar{k}-1=2 \bar{k}<4 \sqrt{\tau},
$$

and we can conclude that

$$
\frac{3 \sqrt{\tau}}{4} \leq R(\tau) \leq 4 \sqrt{\tau}, \quad \tau>16 .
$$


Summing up, we proved that

$$
\begin{aligned}
& N_{A_{1}}(\tau)=\tau+R(\tau), \\
& N_{A_{2}}(\tau)=2 \tau^{1 / 2}+\mathcal{O}(1),
\end{aligned}
$$

where the $R(\tau)$ in (28) satisfies (27). Notice that both $A_{1}$ and $A_{2}$ are elliptic, invertible and positive, so it is possible to consider powers of these operators of arbitrary exponent. Now, we examine separately the three different situations that can arise.

Case $\frac{n_{1}}{m_{1}}>\frac{n_{2}}{m_{2}}$ and $\frac{n_{1}-1}{m_{1}}>\frac{n_{2}}{m_{2}}$

Let us consider the operator

$$
B=A_{1} \otimes A_{2}^{2}
$$

Clearly, $\frac{n_{1}}{m_{1}}=\frac{2}{2}=1>\frac{n_{2}}{m_{2}}=\frac{1}{4}$ and $\frac{n_{1}-1}{m_{1}}=\frac{1}{2}>\frac{n_{2}}{m_{2}}=\frac{1}{4}$, so we are in the first case of Theorem 2, which states that

$$
N_{B}(\tau)=\zeta\left(A_{2}^{2}, 1\right) \tau+\mathcal{O}\left(\tau^{1 / 2}\right)
$$

By Eqs. (23) and (24), we obtain

$$
\begin{aligned}
\sigma(B)= & \left\{\left(k^{2}-k+1\right)\left(n^{2}+1\right)^{2} \mid k, n \in \mathbb{N},\right. \\
& \text { mult } \left.\left(\left(k^{2}-k+1\right)\left(n^{2}+1\right)^{2}\right)=2(2 k+1)\right\} .
\end{aligned}
$$

Therefore,

$$
\begin{aligned}
N_{B}(\tau) & =\sum_{\left(k^{2}-k+1\right)\left(n^{2}+1\right)^{2}<\tau} \sum_{\left(k^{2}-k+1\right)\left(n^{2}+1\right)^{2}<\tau}^{n \in \mathbb{N}, k \in \mathbb{N}, k \in \mathbb{N}} 2(2 k+1) \\
& =2 \sum_{\left(k^{2}-k+1\right)<\frac{\tau}{\left(n^{2}+1\right)^{2}}}^{n \in \mathbb{N}, k \in \mathbb{N}} \operatorname{mult}\left(k^{2}-k+1\right) \\
& =2 \sum_{\left(n^{2}+1\right)^{2}<\tau} N_{A_{1}}\left(\frac{\tau}{\left(n^{2}+1\right)^{2}}\right) \\
& =2\left(\sum_{\left(n^{2}+1\right)^{2}<\tau}^{n \in \mathbb{N}} \frac{\tau}{\left(n^{2}+1\right)^{2}}+R\left(\frac{\tau}{\left(n^{2}+1\right)^{2}}\right)\right) .
\end{aligned}
$$

Notice that in (31) we have made use of (25) to reduce the summation. Let us now show that the estimate (30) is indeed sharp, that is,

$$
\limsup _{\tau \rightarrow+\infty} \frac{\left|N_{B}(\tau)-\zeta\left(A_{2}^{2}, 1\right) \tau\right|}{\tau^{1 / 2}}>0,
$$


by direct computation. In view of (32), we can write

$$
\begin{aligned}
& \limsup _{\tau \rightarrow+\infty} \frac{\left|N_{B}(\tau)-\zeta\left(A_{2}^{2}, 1\right) \tau\right|}{\tau^{1 / 2}} \\
& \quad=\limsup _{\tau \rightarrow+\infty} \frac{\left|2 \sum_{\left(n^{2}+1\right)^{2}<\tau}\left(\frac{\tau}{\left(n^{2}+1\right)^{2}}+R\left(\frac{\tau}{\left(n^{2}+1\right)^{2}}\right)\right)-\zeta\left(A_{2}^{2}, 1\right) \tau\right|}{\tau^{1 / 2}} \\
& \quad=\limsup _{\tau \rightarrow+\infty} \frac{\left|2 \sum_{\left(n^{2}+1\right)^{2}<\tau} \frac{\tau}{\left(n^{2}+1\right)^{2}}-\zeta\left(A_{2}^{2}, 1\right) \tau+2 \sum_{\left(n^{2}+1\right)^{2}<\tau} R\left(\frac{\tau}{\left(n^{2}+1\right)^{2}}\right)\right|}{\tau^{1 / 2}} .
\end{aligned}
$$

We notice that

$$
\limsup _{\tau \rightarrow+\infty} \frac{\left|2 \sum_{\left(n^{2}+1\right)^{2}<\tau} \frac{\tau}{\left(n^{2}+1\right)^{2}}-\zeta\left(A_{2}^{2}, 1\right) \tau\right|}{\tau^{1 / 2}}=\limsup _{\tau \rightarrow+\infty} \tau^{1 / 2}\left(F_{A_{2}^{2}}(\tau, 1)-\zeta\left(A_{2}^{2}, 1\right)\right),
$$

where we have used the notation introduced in Sect. 2. By Proposition $1, F_{A_{2}^{2}}(\tau, 1)-$ $\zeta\left(A_{2}^{2}, 1\right)=\mathcal{O}\left(\tau^{-\frac{3}{4}}\right) ;$ therefore $^{4}$,

$$
\limsup _{\tau \rightarrow+\infty} \frac{\left|2 \sum_{\left(n^{2}+1\right)^{2}<\tau} \frac{\tau}{\left(n^{2}+1\right)^{2}}-\zeta\left(A_{2}^{2}, 1\right) \tau\right|}{\tau^{1 / 2}}=0 .
$$

Since, for all $\tau$,

$$
\sum_{\left(n^{2}+1\right)^{2}<\tau} 2 \frac{\tau}{\left(n^{2}+1\right)^{2}}-\zeta\left(A_{2}^{2}, 1\right) \tau \leq 0
$$

(33) becomes

$$
\begin{aligned}
\limsup _{\tau \rightarrow+\infty} \frac{\left|N_{B}(\tau)-\zeta\left(A_{2}^{2}, 1\right) \tau\right|}{\tau^{1 / 2} \geq} & -\limsup _{\tau \rightarrow+\infty} \frac{\zeta\left(A_{2}^{2}, 1\right)-2 \sum_{\left(n^{2}+1\right)^{2}<\tau} \frac{\tau}{\left.n^{2}+1\right)^{2}}}{\tau^{1 / 2}} \\
& +2 \limsup _{\tau \rightarrow+\infty} \sum_{\left(n^{2}+1\right)^{2}<\tau} \frac{\left|R\left(\frac{\tau}{\left(n^{2}+1\right)^{2}}\right)\right|}{\tau^{1 / 2}} \\
\geq & \frac{3}{2} \limsup _{\tau \rightarrow+\infty} \sum_{\left(n^{2}+1\right)^{2}<\tau} \frac{\tau^{1 / 2}}{\left(n^{2}+1\right) \tau^{1 / 2}} \\
= & \frac{3}{2} \zeta\left(A_{2}^{2}, \frac{1}{2}\right) .
\end{aligned}
$$

Here, we have used the estimates (27), and the quantities $\frac{n_{1}}{m_{1}}=1$ and $\frac{n_{1}-1}{n_{2}}=\frac{1}{2}$ are larger than $\frac{n_{2}}{m_{2}}=\frac{1}{4}$. The latter implies that $\zeta\left(A_{2}^{2}, \frac{1}{2}\right)$ is a finite, positive quantity ${ }^{5}$, in view of the holomorphic properties of the spectral $\zeta$-function of elliptic positive pseudodifferential operators on closed manifolds, see [25]. This proves the desired result.

${ }^{4}$ Actually, here one could prove directly that $F_{1}(\tau)-\zeta\left(A_{2}^{2}, 1\right)$ is asymptotic to $\tau^{-\frac{3}{4}}$.

5 The convergence of the involved series is straightforward. 
Case $\frac{n_{1}}{m_{1}}>\frac{n_{2}}{m_{2}}$ and $\frac{n_{1}-1}{m_{1}}=\frac{n_{2}}{m_{2}}$

We consider the operator

$$
C=A_{1} \otimes A_{2}
$$

Clearly, $\frac{n_{1}}{m_{1}}=\frac{2}{2}=1>\frac{n_{2}}{m_{2}}=\frac{1}{2}$ and $\frac{n_{1}-1}{m_{1}}=\frac{1}{2}=\frac{n_{2}}{m_{2}}$ so that we are in second case of Theorem 2, which now states that

$$
N_{C}(\tau)=\zeta\left(A_{2}, 1\right) \tau+\mathcal{O}\left(\tau^{1 / 2} \log \tau\right)
$$

Using (23) and (24), we obtain explicitly the spectrum of $C$, namely

$$
\sigma(C)=\left\{\left(k^{2}-k+1\right)\left(n^{2}+1\right) \mid \text { mult }\left(\left(k^{2}-k+1\right)\left(n^{2}+1\right)\right)=2(2 k+1)\right\} .
$$

Therefore, using (25),

$$
\begin{aligned}
N_{C}(\tau) & =\sum_{\left(k^{2}-k+1\right)\left(n^{2}+1\right)<\tau}^{n \in \mathbb{N}, k \in \mathbb{N}} 2(2 k+1) \\
& =2 \sum_{\left(k^{2}-k+1\right)<\frac{\tau}{n^{2}+1}}^{n \in \mathbb{N}, k \in \mathbb{N}} \text { mult }\left(k^{2}+k+1\right) \\
& =2 \sum_{\left(n^{2}+1\right)<\tau}^{n \in \mathbb{N}} N_{A_{1}}\left(\frac{\tau}{n^{2}+1}\right) \\
& =2 \sum_{n^{2}+1<\tau}^{\sum_{n}}\left(\frac{\tau}{n^{2}+1}+R\left(\frac{\tau}{n^{2}+1}\right)\right) .
\end{aligned}
$$

Let us check directly that

$$
\limsup _{\tau \rightarrow+\infty} \frac{\left|N_{C}(\tau)-\zeta\left(A_{2}, 1\right) \tau\right|}{\tau^{1 / 2} \log \tau}>0 .
$$

Using (34) and (27), we can write

$$
\begin{aligned}
\limsup _{\tau \rightarrow+\infty} \frac{\left|N_{C}(\tau)-\zeta\left(A_{2}, 1\right) \tau\right|}{\tau^{1 / 2} \log \tau}= & \limsup _{\tau \rightarrow+\infty} \frac{\left|2 \sum_{n^{2}+1<\tau}\left(\frac{\tau}{n^{2}+1}+R\left(\frac{\tau}{n^{2}+1}\right)\right)-\zeta\left(A_{2}, 1\right) \tau\right|}{\tau^{1 / 2} \log \tau} \\
\geq & -\limsup _{\tau \rightarrow+\infty} \frac{\tau^{1 / 2}\left(\zeta\left(A_{2}, 1\right)-2 \sum_{n^{2}+1<\tau} \frac{1}{n^{2}+1}\right)}{\log \tau} \\
& +\limsup _{\tau \rightarrow+\infty} \frac{3}{4} \tau^{1 / 2} \frac{2 \sum_{n^{2}+1<\tau} \frac{1}{\left(n^{2}+1\right)^{1 / 2}}}{\tau^{1 / 2} \log \tau} \\
\geq & -\limsup _{\tau \rightarrow+\infty} \tau^{\frac{1}{2}} \frac{2 \sum_{n^{2}+1 \geq \tau} \frac{1}{n^{2}+1}}{\log \tau}+\limsup _{\tau \rightarrow+\infty} \frac{3}{2} \frac{\sum_{n^{2}+1<\tau} \frac{1}{\left(n^{2}+1\right)^{1 / 2}}}{\log \tau}
\end{aligned}
$$


Finally, using the results of Proposition 1 (or directly, by integral inequalities), we obtain that

$$
\limsup _{\tau \rightarrow+\infty} \tau^{\frac{1}{2}} \frac{2 \sum_{n^{2}+1 \geq \tau} \frac{1}{n^{2}+1}}{\log \tau}=\lim _{\tau \rightarrow+\infty} \tau^{\frac{1}{2}} \frac{2 \sum_{n^{2}+1 \geq \tau} \frac{1}{n^{2}+1}}{\log \tau}=0 .
$$

Moreover,

$$
\limsup _{\tau \rightarrow+\infty} \frac{3}{2} \frac{\sum_{n^{2}+1<\tau} \frac{1}{n^{2}+1}}{\log \tau}=\frac{3}{4},
$$

so that, by means of (36), the desired result is proven also in this second case.

Case $\frac{n_{1}}{m_{1}}>\frac{n_{2}}{m_{2}}$ and $\frac{n_{1}-1}{m_{1}}<\frac{n_{2}}{m_{2}}$

In this situation, we consider the operator

$$
D=A_{1} \otimes A_{2}^{\frac{3}{4}} .
$$

Clearly, $\frac{n_{1}}{m_{1}}=\frac{2}{2}=1>\frac{n_{2}}{m_{2}}=\frac{2}{3}$ and $\frac{n_{1}-1}{m_{1}}=\frac{1}{2}<\frac{n_{2}}{m_{2}}=\frac{2}{3}$, so we are in the third case of Theorem 2, which implies that

$$
N_{D}(\tau)=\zeta\left(A_{2}^{\frac{3}{4}}, 1\right) \tau+\mathcal{O}\left(\tau^{\frac{2}{3}}\right) .
$$

It is immediate to observe that

$$
\begin{aligned}
\sigma(D)= & \left\{\left(k^{2}+k+1\right)\left(n^{2}+1\right)^{3 / 4} \mid\right. \\
& \text { mult } \left.\left(\left(k^{2}+k+1\right)\left(n^{2}+1\right)^{3 / 4}\right)=2(2 k+1)\right\} .
\end{aligned}
$$

Therefore, using again (25), we obtain

$$
\begin{aligned}
N_{D}(\tau) & =\sum_{\left(k^{2}-k+1\right)\left(n^{2}+1\right)^{3 / 4}<\tau}^{n \in \mathbb{N}, k \in \mathbb{N}} 2(2 k+1) \\
& =2 \sum_{\left(k^{2}-k+1\right)<\frac{\tau}{\left(n^{2}+1\right)^{3 / 4}}}^{n \in \mathbb{N}, k \in \mathbb{N}} \text { mult }\left(k^{2}-k+1\right) \\
& =2 \sum_{\left(n^{2}+1\right)^{3 / 4}<\tau}^{n \in \mathbb{N}} N_{A_{1}}\left(\frac{\tau}{\left(n^{2}+1\right)^{3 / 4}}\right) \\
& =2 \sum_{\left(n^{2}+1\right)^{3 / 4}<\tau}^{n \in \mathbb{N}}\left(\frac{\tau}{\left(n^{2}+1\right)^{3 / 4}}+R\left(\frac{\tau}{\left(n^{2}+1\right)^{3 / 4}}\right)\right) .
\end{aligned}
$$

Let us now compute directly

$$
\limsup _{\tau \rightarrow+\infty} \frac{\left|N_{D}(\tau)-\zeta\left(A_{2}^{3 / 4}, 1\right) \tau\right|}{\tau^{2 / 3}} .
$$


By (39), we find

$$
\begin{aligned}
& \limsup _{\tau \rightarrow+\infty} \frac{\left|N_{D}(\tau)-\zeta\left(A_{2}^{3 / 4}, 1\right) \tau\right|}{\tau^{2 / 3}} \\
& =\limsup _{\tau \rightarrow+\infty} \frac{\left|2 \sum_{\left(n^{2}+1\right)^{3 / 4}<\tau}\left(\frac{\tau}{\left(n^{2}+1\right)^{3 / 4}}+R\left(\frac{\tau}{\left(n^{2}+1\right)^{\frac{3}{4}}}\right)\right)-\zeta\left(A_{2}^{3 / 4}, 1\right) \tau\right|}{\tau^{2 / 3}} \\
& =\limsup _{\tau \rightarrow+\infty} \tau^{-2 / 3} \cdot \mid 2 \sum_{\left(n^{2}+1\right)^{3 / 4}<\tau} \frac{\tau}{\left(n^{2}+1\right)^{3 / 4}}-\zeta\left(A_{2}^{3 / 4}, 1\right) \tau+ \\
& \quad R\left(\frac{\tau}{\left(n^{2}+1\right)^{\frac{3}{4}}}\right) \mid .
\end{aligned}
$$

We also notice that

$$
\begin{aligned}
& \lim _{\tau \rightarrow+\infty} \frac{\left|2 \sum_{\left(n^{2}+1\right)^{3 / 4}<\tau} \frac{\tau}{\left(n^{2}+1\right)^{3 / 4}}-\zeta\left(A_{2}^{3 / 4}, 1\right) \tau\right|}{\tau^{2 / 3}} \\
& =\lim _{\tau \rightarrow+\infty} \frac{\zeta\left(A_{2}^{3 / 4}, 1\right) \tau-2 \sum_{\left(n^{2}+1\right)^{3 / 4}<\tau} \frac{\tau}{\left(n^{2}+1\right)^{3 / 4}}}{\tau^{2 / 3}} \\
& =\lim _{\tau \rightarrow+\infty} 2 \tau^{1 / 3} \sum_{\left(n^{2}+1\right)^{3 / 4} \geq \tau} \frac{1}{\left(n^{2}+1\right)^{3 / 4}},
\end{aligned}
$$

and that

$$
\sum_{(n+1)^{3 / 2} \geq \tau} \frac{1}{(n+1)^{3 / 2}} \leq \sum_{\left(n^{2}+1\right)^{3 / 4} \geq \tau} \frac{1}{\left(n^{2}+1\right)^{3 / 4}} \leq \sum_{n^{3 / 2} \geq \tau} \frac{1}{n^{3 / 2}} .
$$

Using the standard integral criteria of series convergence, one can easily check that

$$
\lim _{\tau \rightarrow+\infty} \tau^{1 / 3} \sum_{(n+1)^{3 / 2} \geq \tau} \frac{1}{(n+1)^{3 / 2}}=\lim _{\tau \rightarrow+\infty} \tau^{1 / 3} \sum_{n^{3 / 2} \geq \tau} \frac{1}{n^{3 / 2}}=2 .
$$

Hence,

$$
\lim _{\tau \rightarrow+\infty} 2 \tau^{1 / 3} \sum_{\left(n^{2}+1\right)^{3 / 4} \geq \tau} \frac{1}{\left(n^{2}+1\right)^{3 / 4}}=4
$$

By a similar argument, we also have that

$$
\lim _{\tau \rightarrow+\infty} \tau^{-1 / 6} \sum_{\left(n^{2}+1\right)^{3 / 4}<\tau} \frac{1}{\left(n^{2}+1\right)^{3 / 8}}=4
$$


In view of (27), (40) and (41) we finally obtain

$$
\begin{aligned}
\limsup _{\tau \rightarrow+\infty} \frac{\left|N_{D}(\tau)-\zeta\left(A_{2}^{3 / 4}, 1\right) \tau\right|}{\tau^{2 / 3}} \geq & \limsup _{\tau \rightarrow+\infty} \frac{N_{D}(\tau)-\zeta\left(A_{2}^{3 / 4}, 1\right) \tau}{\tau^{2 / 3}} \\
= & -\lim _{\tau \rightarrow+\infty} 2 \tau^{1 / 3} \sum_{\left(n^{2}+1\right)^{3 / 4} \geq \tau} \frac{1}{\left(n^{2}+1\right)^{3 / 4}} \\
& +\limsup _{\tau \rightarrow+\infty} 2 \frac{\sum_{\left(n^{2}+1\right)^{3 / 4}<\tau} R\left(\frac{\tau}{\left(n^{2}+1\right)^{3 / 4}}\right)}{\tau^{2 / 3}} \\
\geq & -4+\frac{3}{2} \limsup _{\tau \rightarrow+\infty} \tau^{-1 / 6} \sum_{\left(n^{2}+1\right)^{3 / 4}<\tau} \frac{1}{\left(n^{2}+1\right)^{3 / 8}} \\
\geq & -4+6=2>0 .
\end{aligned}
$$

Equation (42) proves the desired result also in this last case.

Acknowledgments We wish to thank L. Rodino, S. Pilipović, F. Nicola, and J. Seiler, for useful discussions and comments. The first author has been supported by the Gruppo Nazionale per l'Analisi Matematica, la Probabilità e le loro Applicazioni (GNAMPA) of the Istituto Nazionale di Alta Matematica (INdAM). The second and third author have been partially supported by the Gruppo Nazionale per l'Analisi Matematica, la Probabilità e le loro Applicazioni (GNAMPA) of the Istituto Nazionale di Alta Matematica (INdAM).

\section{Appendix: The Dirichlet divisors problem}

Counting functions of the type (2) suggest a spectral approach to a prominent type of lattice problem, the so-called Dirichlet divisors problem. Let us suppose that the spectrum of both $A_{1}$ and $A_{2}$ in (2) is formed by all strictly positive natural numbers, each with multiplicity one. Then,

$$
N_{A}(\tau)=\sum_{n \cdot m<\tau} 1=D(\tau)
$$

The function $D(\tau)$ is called Dirichlet divisor summatory function, and it is straightforward to check that it amounts the number of points with integer coordinates belonging to the first quadrant of the Cartesian plane which lie below the hyperbola $x y=\tau$. In 1849, Dirichlet proved that

$$
D(\tau)=\tau \log \tau+(2 \gamma-1) \tau+\mathcal{O}\left(\tau^{1 / 2}\right),
$$

where $\gamma$ is the Euler-Mascheroni constant, namely

$$
\gamma=\lim _{\tau \rightarrow+\infty}\left(\sum_{0<n<\tau} \frac{1}{n}-\int_{0}^{\tau} \frac{1}{x} \mathrm{~d} x\right),
$$

or, equivalently,

$$
\gamma=\lim _{z \rightarrow 1}(z-1) \zeta_{R}(z)
$$

where $\zeta_{R}(z)$ is the Riemann $\zeta$-function. Several papers aimed at finding the sharp remainder term in (43), see [19] for an overview on this type of problems. Hardy, in [14], proved that 
$\mathcal{O}\left(\tau^{\frac{1}{4}}\right)$ is a lower bound for the remainder in (43). It is conjectured that the sharp estimate in this case is $\mathcal{O}\left(\tau^{\frac{1}{4}+\epsilon}\right)$ or, more precisely, $\mathcal{O}\left(\tau^{1 / 4} \log \tau\right)$. The best-known result, due to Huxley, is that the remainder is $\mathcal{O}\left(\tau^{\alpha}(\log \tau)^{\beta+1}\right)$, where

$$
\alpha=\frac{131}{416} \sim 0,3149 \ldots \quad \beta=\frac{18627}{8320} \sim 2,2513 \ldots
$$

In order to have a spectral interpretation of the Dirichlet divisor problem, a global bisingular calculus based on Shubin calculus has been introduced in [6]. Then, the following Hermite-type operator

$$
H_{j}=\frac{1}{2}\left(-\partial_{x_{j}}^{2}+x_{j}^{2}\right)+\frac{1}{2}, \quad j=1,2,
$$

has been examined. Using Hermite polynomials, it turns out that $\sigma\left(H_{j}\right)=\{n\}_{n \in \mathbb{N}^{*}}, j=1,2$, and each eigenvalue has multiplicity one. Therefore, $\sigma\left(H_{1} \otimes H_{2}\right)=\{n \cdot m\}_{(n, m) \in\left(\mathbb{N}^{*}\right)^{2}}$ and

$$
N_{H_{1} \otimes H_{2}}(\tau)=D(\tau) .
$$

This clear spectral meaning of the Dirichlet divisor problem was one of the main motivation of the papers $[6,11]$. For the connection between Dirichlet divisor problem and standard bisingular operators on the product of closed manifolds, see [4]. Actually, since we deal with the non-symmetric case, it is not possible to attack directly the traditional Dirichlet divisor problem through the approach described in the previous sections, while our techniques are well suited to treat generalized anisotropic Dirichlet divisors problems such as, for instance,

$$
N_{H_{1}^{\alpha} \otimes H_{2}^{\beta}}(\tau)=\sum_{n^{\alpha} \cdot m^{\beta}<\tau} 1, \quad \alpha \neq \beta .
$$

In [11], it is proven that

$$
N_{H_{1}^{\alpha} \otimes H_{2}^{\beta}}(\tau)=\zeta\left(\frac{\alpha}{\beta}\right) \tau^{\frac{1}{\beta}}+\zeta\left(\frac{\beta}{\alpha}\right) \tau^{\frac{1}{\alpha}}+\mathcal{O}\left(\tau^{\frac{1}{\alpha+\beta}}\right),
$$

where $\zeta$ is the meromorphic continuation of the Riemann $\zeta$-function. Notice that (44) proves the sharpness of the result stated in Theorem 3 in the case $\frac{2 n_{2}}{m_{2}}>\frac{2 n_{1}-1}{m_{1}}$.

\section{References}

1. Aramaki, J.: On an extension of the Ikehara Tauberian theorem. Pac. J. Math. 133(1), 13-30 (1988)

2. Arendt, W., Nittka, R., Peter, W., Steiner, F.: Weyl's Law: Spectral Properties of the Laplacian in Mathematics and Physics. In: Mathematical Analysis of Evolution, Information, and Complexity. Wiley-VCH Verlag GmbH \& Co. KGaA, Weinheim (2009)

3. Atiyah, M.F., Singer, I.M.: The index of elliptic operators. I. Ann. Math. 87(2), 484-530 (1968)

4. Battisti, U.: Weyl asymptotics of bisingular operators and Dirichlet divisor problem. Math. Z. 272(3-4), 1365-1381 (2012)

5. Battisti, U., Coriasco, S.: Wodzicki residue for operators on manifolds with cylindrical ends. Ann. Glob. Anal. Geom. 40(2), 223-249 (2011)

6. Battisti, U., Gramchev, T., Rodino, L., Pilipović, S.: Globally bisingular elliptic operators. In: Operator Theory, Pseudo-Differential Equations, and Mathematical Physics, Operator Theory: Advances and Applications, vol. 228, pp. 21-38. Birkhäuser/Springer, Basel (2013)

7. Boggiatto, P., Nicola, F.: Non-commutative residues for anisotropic pseudo-differential operators in $\mathbb{R}^{n}$. J. Funct. Anal. 203(2), 305-320 (2003)

8. Coriasco, S., Maniccia, L.: On the spectral asymptotics of operators on manifolds with ends. Abstr. Appl. Anal. 2013, 909782 (2013) 
9. Datchev, K., Dyatlov, S.: Fractal Weyl laws for asymptotically hyperbolic manifolds. Geom. Funct. Anal. 23(4), 1145-1206 (2013)

10. Gil, J.B., Loya, P.A.: On the noncommutative residue and the heat trace expansion on conic manifolds. Manuscr. Math. 109(3), 309-327 (2002)

11. Gramchev, T., Pilipović, S., Rodino, L., Vindas, J.: Weyl asymptotics for tensor products of operators and Dirichlet divisors. Ann. Mat. Pura Appl. (2014). doi:10.1007/s10231-014-0400-z

12. Grigis, A., Sjöstrand, J.: Microlocal analysis for differential operators. An introduction. In: London Mathematical Society Lecture Note Series, vol. 196. Cambridge University Press, Cambridge (1994)

13. Guillemin, V.: A new proof of Weyl's formula on the asymptotic distribution of eigenvalues. Adv. Math. 55(2), 131-160 (1985)

14. Hardy, G.H.: On Dirichlet's divisor problem. Proc. London Math. Soc. 15(2), 1-25 (1916)

15. Hörmander, L.: The spectral function of an elliptic operator. Acta Math. 121, 193-218 (1968)

16. Hörmander, L.: The analysis of linear partial differential operators IV: Fourier integral operators. In: Classics in Mathematics, Springer, Berlin, Reprint of the 1994 edition (2007)

17. Helffer, B.: Théorie spectrale pour des opérateurs globalement elliptiques, Astérisque (112). Société Mathématique de France, Paris (1984)

18. Helffer, B., Robert, D.: Comportement asymptotique précise du spectre d'opérateurs globalement elliptiques dans $\mathbf{R}^{n}$, Goulaouic-Meyer-Schwartz Seminar, 1980-1981, École Polytech., Palaiseau, pp. Exp. No. II, 23 (1981)

19. Ivić, A., Krätzel, E., Kühleitner, M., Nowak, W.G.: Lattice points in large regions and related arithmetic functions: recent developments in a very classic topic. In: Elementare und analytische Zahlentheorie, Schr. Wiss. Ges. Johann Wolfgang Goethe Univ. Frankfurt am Main, 20, Franz Steiner Verlag Stuttgart, Stuttgart, pp. 89-128 (2006)

20. Moroianu, S.: Weyl laws on open manifolds. Math. Ann. 340(1), 1-21 (2008)

21. Nicola, F.: Trace functionals for a class of pseudo-differential operators in $\mathbb{R}^{n}$. Math. Phys. Anal. Geom. 6(1), 89-105 (2003)

22. Nicola, F., Rodino, L.: Residues and index for bisingular operators, $C^{*}$-algebras and elliptic theory, pp. 187-202. In: Trends Math., Birkhäuser, Basel (2006)

23. Rodino, L.: A class of pseudo differential operators on the product of two manifolds and applications. Ann. Scuola Norm. Sup. Pisa Cl. Sci. (4) 2(2), 287-302 (1975)

24. Safarov, Y., Vassiliev, D.: The asymptotic distribution of eigenvalues of partial differential operators. In: Translations of Mathematical Monographs. vol. 155, American Mathematical Society, Providence, RI, Translated from the Russian manuscript by the authors (1997)

25. Seeley, R.T.: Complex powers of an elliptic operator. In: Singular Integrals (Proceedings Symposium Pure Mathematics, Chicago, Ill., 1966), pp. 288-307. American Mathematical Society, Providence, RI (1967)

26. Shubin, M.A.: Pseudodifferential Operators and Spectral Theory. Springer, Berlin (2001) 